\title{
Functions of Baire class one
}

\author{
by \\ Denny H. Leung and Wee-Kee Tang (Singapore)
}

\begin{abstract}
Let $K$ be a compact metric space. A real-valued function on $K$ is said to be of Baire class one (Baire-1) if it is the pointwise limit of a sequence of continuous functions. We study two well known ordinal indices of Baire- 1 functions, the oscillation index $\beta$ and the convergence index $\gamma$. It is shown that these two indices are fully compatible in the following sense: a Baire-1 function $f$ satisfies $\beta(f) \leq \omega^{\xi_{1}} \cdot \omega^{\xi_{2}}$ for some countable ordinals $\xi_{1}$ and $\xi_{2}$ if and only if there exists a sequence $\left(f_{n}\right)$ of Baire- 1 functions converging to $f$ pointwise such that $\sup _{n} \beta\left(f_{n}\right) \leq \omega^{\xi_{1}}$ and $\gamma\left(\left(f_{n}\right)\right) \leq \omega^{\xi_{2}}$. We also obtain an extension result for Baire-1 functions analogous to the Tietze Extension Theorem. Finally, it is shown that if $\beta(f) \leq \omega^{\xi_{1}}$ and $\beta(g) \leq \omega^{\xi_{2}}$, then $\beta(f g) \leq \omega^{\xi}$, where $\xi=\max \left\{\xi_{1}+\xi_{2}, \xi_{2}+\xi_{1}\right\}$. These results do not assume the boundedness of the functions involved.
\end{abstract}

1. Preliminaries. Let $K$ be a compact metric space. A function $f$ : $K \rightarrow \mathbb{R}$ is said to be of Baire class one, or simply Baire-1, if there exists a sequence $\left(f_{n}\right)$ of real-valued continuous functions that converges pointwise to $f$. Let $\mathfrak{B}_{1}(K)$ (respectively, $\mathcal{B}_{1}(K)$ ) be the set of all real-valued (respectively, bounded real-valued) Baire-1 functions on $K$. Several authors have studied Baire-1 functions in terms of ordinal ranks associated to each function. (See, e.g., [2], [3] and [4].) In this paper, we study the relationship between two of these ordinal ranks, namely the oscillation rank $\beta$ and the convergence rank $\gamma$.

We begin by recalling the definitions of the indices $\beta$ and $\gamma$. Suppose that $H$ is a compact metric space, and $f$ is a real-valued function whose domain contains $H$. For any $\varepsilon>0$, let $H^{0}(f, \varepsilon)=H$. If $H^{\alpha}(f, \varepsilon)$ is defined for some countable ordinal $\alpha$, let $H^{\alpha+1}(f, \varepsilon)$ be the set of all those $x \in H^{\alpha}(f, \varepsilon)$ such that for every open set $U$ containing $x$, there are two points $x_{1}$ and $x_{2}$ in $U \cap H^{\alpha}(f, \varepsilon)$ with $\left|f\left(x_{1}\right)-f\left(x_{2}\right)\right| \geq \varepsilon$. For a countable limit ordinal $\alpha$, we let

$$
H^{\alpha}(f, \varepsilon)=\bigcap_{\alpha^{\prime}<\alpha} H^{\alpha^{\prime}}(f, \varepsilon) .
$$

2000 Mathematics Subject Classification: Primary 26A21; Secondary 03E15, 54C30. Key words and phrases: Baire-1 functions, convergence index, oscillation index. 
The index $\beta_{H}(f, \varepsilon)$ is taken to be the least $\alpha$ with $H^{\alpha}(f, \varepsilon)=\emptyset$ if such an $\alpha$ exists, and $\omega_{1}$ otherwise. The oscillation index of $f$ is

$$
\beta_{H}(f)=\sup \left\{\beta_{H}(f, \varepsilon): \varepsilon>0\right\} .
$$

If the ambient space $H$ is clear from the context, we write $\beta(f, \varepsilon)$ and $\beta(f)$ in place of $\beta_{H}(f, \varepsilon)$ and $\beta_{H}(f)$ respectively.

The $\gamma$ index is defined analogously. If $\left(f_{n}\right)$ is a sequence of real-valued functions such that $H \subseteq \bigcap_{n} \operatorname{dom}\left(f_{n}\right)$, let $H^{0}\left(\left(f_{n}\right), \varepsilon\right)=H$ for any $\varepsilon>0$. If $H^{\alpha}\left(\left(f_{n}\right), \varepsilon\right)$ has been defined for some countable ordinal $\alpha$, let $H^{\alpha+1}\left(\left(f_{n}\right), \varepsilon\right)$ be the set of all those $x \in H^{\alpha}\left(\left(f_{n}\right), \varepsilon\right)$ such that for every open set $U$ containing $x$ and any $m \in \mathbb{N}$, there are two integers $n_{1}, n_{2}$ with $n_{1}>n_{2}>m$ and $x^{\prime} \in U \cap H^{\alpha}\left(\left(f_{n}\right), \varepsilon\right)$ such that $\left|f_{n_{1}}\left(x^{\prime}\right)-f_{n_{2}}\left(x^{\prime}\right)\right| \geq \varepsilon$. Define

$$
H^{\alpha}\left(\left(f_{n}\right), \varepsilon\right)=\bigcap_{\alpha^{\prime}<\alpha} H^{\alpha^{\prime}}\left(\left(f_{n}\right), \varepsilon\right)
$$

if $\alpha$ is a countable limit ordinal. Let $\gamma_{H}\left(\left(f_{n}\right), \varepsilon\right)$ be the least $\alpha$ with $H^{\alpha}\left(\left(f_{n}\right), \varepsilon\right)=\emptyset$ if such an $\alpha$ exists, and $\omega_{1}$ otherwise. Finally, the convergence index of $\left(f_{n}\right)$ is the ordinal

$$
\gamma_{H}\left(\left(f_{n}\right)\right)=\sup \left\{\gamma_{H}\left(\left(f_{n}\right), \varepsilon\right): \varepsilon>0\right\} .
$$

Again, if there is no ambiguity about the space $H$, we write $\gamma\left(\left(f_{n}\right), \varepsilon\right)$ and $\gamma\left(\left(f_{n}\right)\right)$ for $\gamma_{H}\left(\left(f_{n}\right), \varepsilon\right)$ and $\gamma_{H}\left(\left(f_{n}\right)\right)$ respectively.

It is known that a function $f: K \rightarrow \mathbb{R}$ is Baire- 1 if and only if $\beta(f)<\omega_{1}$. (See [3, Proposition 1.2].) Following [3], we define the set of functions of small Baire class $\xi$ and the set of bounded functions of small Baire class $\xi$ for each countable ordinal $\xi$ as

$$
\mathfrak{B}_{1}^{\xi}(K)=\left\{f \in \mathfrak{B}_{1}(K): \beta(f) \leq \omega^{\xi}\right\}
$$

and

$$
\mathcal{B}_{1}^{\xi}(K)=\left\{f \in \mathcal{B}_{1}(K): \beta(f) \leq \omega^{\xi}\right\}
$$

respectively. In [4], the following results are shown.

TheORem 1.1. Let $K$ be a compact metric space.

(a) [4, Theorem 7] If $\xi$ is a finite ordinal, then a function $f$ is in $\mathcal{B}_{1}^{\xi+1}(K)$ if and only if there exists a sequence $\left(f_{n}\right)$ in $\mathcal{B}_{1}^{1}(K)$ converging pointwise to $f$ such that $\gamma\left(\left(f_{n}\right)\right) \leq \omega^{\xi}$.

(b) [4, Corollary 9] If $\xi$ is an infinite countable ordinal, and $f \in \mathcal{B}_{1}(K)$ is the pointwise limit of a sequence $\left(f_{n}\right)$ in $\mathcal{B}_{1}^{1}(K)$ such that $\gamma\left(\left(f_{n}\right)\right) \leq \omega^{\xi}$, then $\beta(f) \leq \omega^{\xi}$.

One of our main results generalizes and unifies the two parts of Theorem 1.1. 
THEOREM 1.2. Let $K$ be a compact metric space and let $\xi_{1}, \xi_{2}$ be countable ordinals. A function $f$ is in $\mathfrak{B}_{1}^{\xi_{1}+\xi_{2}}(K)$, respectively, $\mathcal{B}_{1}^{\xi_{1}+\xi_{2}}(K)$, if and only if there exists a sequence $\left(f_{n}\right)$ in $\mathfrak{B}_{1}^{\xi_{1}}(K)$, respectively, a bounded sequence $\left(f_{n}\right)$ in $\mathcal{B}_{1}^{\xi_{1}}(K)$, converging pointwise to $f$ such that $\gamma\left(\left(f_{n}\right)\right) \leq \omega^{\xi_{2}}$.

In the course of proving Theorem 1.2, we show that any Baire-1 function $f$ on a closed subspace $H$ of a compact metric space $K$ can be extended to a Baire-1 function $g$ on $K$ such that $\beta_{H}(f)=\beta_{K}(g)$ (Theorem 3.6). When $\beta_{H}(f)=1$, this is the familiar Tietze Extension Theorem. Proposition 2.1 and Theorem 2.3 in [3] imply that for a bounded Baire-1 function $f, \beta(f)$ is the smallest ordinal $\xi$ such that there exists a sequence $\left(f_{n}\right)$ of continuous functions converging pointwise to $f$ and having $\gamma\left(\left(f_{n}\right)\right)=\xi$. Theorem 5.5 below shows that the same result holds without the boundedness assumption on the function $f$. In the last section, we consider the product of Baire-1 functions. In contrast to the class $\mathcal{B}_{1}^{\xi}(K)$, the class $\mathfrak{B}_{1}^{\xi}(K)$ is not closed under multiplication. Theorem 6.5 shows that if $f \in \mathfrak{B}_{1}^{\xi_{1}}(K)$ and $g \in \mathfrak{B}_{1}^{\xi_{2}}(K)$, then $f g \in \mathfrak{B}_{1}^{\xi}(K)$, where $\xi=\max \left\{\xi_{1}+\xi_{2}, \xi_{2}+\xi_{1}\right\}$. It is also shown that this result is the best possible.

Our notation is standard. In what follows, $K$ will always denote a compact metric space. If $H$ is a closed subset of $K$, the derived set $H^{\prime}$ is the set of all limit points of $H$. A transfinite sequence of derived sets is defined in the usual manner. Let $H^{(0)}=H$ and $H^{(\alpha+1)}=\left(H^{(\alpha)}\right)^{\prime}$ for any ordinal $\alpha$. If $\alpha$ is a limit ordinal, let

$$
H^{(\alpha)}=\bigcap_{\alpha^{\prime}<\alpha} H^{\left(\alpha^{\prime}\right)} .
$$

It is easy to observe that $H^{\alpha}(f, \varepsilon) \subseteq H^{(\alpha)}$ and $H^{\alpha}\left(\left(f_{n}\right), \varepsilon\right) \subseteq H^{(\alpha)}$, where $H^{\alpha}(f, \varepsilon)$ and $H^{\alpha}\left(\left(f_{n}\right), \varepsilon\right)$ are the sets associated with the oscillation index and the convergence index respectively. Given real-valued functions $f$ and $g$ defined on a set $S$, we let

$$
\|f-g\|_{S}=\sup \{|f(s)-g(s)|: s \in S\} .
$$

When there is no cause for confusion, we write $\|f-g\|$ for $\|f-g\|_{S}$. Since we shall be dealing with unbounded functions in general, this functional can take the value $\infty$ and is not a "norm". However, it is compatible with the topology of uniform convergence on the set $\mathbb{R}^{S}$ of all real-valued functions on $S$ in the sense that the sets

$$
U(f, \varepsilon)=\left\{g:\|g-f\|_{S}<\varepsilon\right\}
$$

form a basis for the said topology.

Acknowledgements. We thank the referee for pointing out an error in a previous version of the proof of Theorem 6.9. 
2. Oscillation and convergence of Baire-1 functions. We begin by proving a result that yields an upper bound of the oscillation index of a Baire- 1 function $f$ as the product of the convergence index of a sequence of functions $\left(f_{n}\right)$ converging pointwise to $f$, and the supremum of the oscillation indices of $f_{n}$ 's.

Lemma 2.1. Let $U$ and $L$ be sets such that $U \subseteq L \subseteq K$, where $U$ is open in $K$ and $L$ is closed in $K$. Suppose $f, f_{n}(n \geq 1)$ are Baire-1 functions on $K, \alpha<\omega_{1}$, and $\varepsilon>0$. Then

(a) $L^{\alpha}(f, \varepsilon) \subseteq K^{\alpha}(f, \varepsilon) \cap L$

(b) $L^{\alpha}\left(\left(f_{n}\right), \varepsilon\right) \subseteq K^{\alpha}\left(\left(f_{n}\right), \varepsilon\right) \cap L$,

(c) $K^{\alpha}(f, \varepsilon) \cap U \subseteq L^{\alpha}(f, \varepsilon)$,

(d) $K^{\alpha}\left(\left(f_{n}\right), \varepsilon\right) \cap U \subseteq L^{\alpha}\left(\left(f_{n}\right), \varepsilon\right)$.

Proof. We only prove (c). The proof is by induction on $\alpha$. The statement is trivial if $\alpha=0$ or a limit ordinal. Suppose the statement is true for all ordinals not greater than $\alpha$. Let $x \in K^{\alpha+1}(f, \varepsilon) \cap U$. If $N$ is a neighborhood of $x$ in $K$, then $N \cap U$ is open in $K$. Thus there exist $x_{1}, x_{2} \in(N \cap U) \cap$ $K^{\alpha}(f, \varepsilon)=N \cap\left(U \cap K^{\alpha}(f, \varepsilon)\right) \subseteq N \cap L^{\alpha}(f, \varepsilon)$ such that $\left|f\left(x_{1}\right)-f\left(x_{2}\right)\right| \geq \varepsilon$. Hence $x \in L^{\alpha+1}(f, \varepsilon)$.

Proposition 2.2. Let $\left(f_{n}\right)$ be a sequence in $\mathfrak{B}_{1}(K)$ and let $\varepsilon>0$. Suppose that $\beta\left(f_{n}, \varepsilon\right) \leq \beta_{0}$ for all $n \in \mathbb{N}$, and $\gamma\left(\left(f_{n}\right), \varepsilon\right) \leq \gamma_{0}$. If $\left(f_{n}\right)$ converges pointwise to a function $f$, then $\beta(f, 3 \varepsilon) \leq \beta_{0} \cdot \gamma_{0}$.

Proof. We first consider the case $\gamma_{0}=1$. Then $K^{1}\left(\left(f_{n}\right), \varepsilon\right)=\emptyset$. For each $x \in K$, there exist an open neighborhood $U_{x}$ of $x$ and $p_{x} \in \mathbb{N}$ such that whenever $n>m>p_{x}$,

$$
\left|f_{n}\left(x^{\prime}\right)-f_{m}\left(x^{\prime}\right)\right|<\varepsilon
$$

for all $x^{\prime} \in U_{x}$. By the compactness of $K$, there exist $x_{1}, \ldots, x_{k}$ such that

$$
K \subseteq \bigcup_{i=1}^{k} U_{x_{i}} .
$$

Let $p_{0}=\max \left\{p_{x_{1}}, \ldots, p_{x_{k}}\right\}$. Then for all $n>m>p_{0}$ and $y \in K$, we have $y \in U_{x_{i}}$ for some $i, 1 \leq i \leq k$. Since $n>m>p_{x_{i}}$,

$$
\left|f_{n}(y)-f_{m}(y)\right|<\varepsilon .
$$

Taking the limit as $n \rightarrow \infty$, we have

$$
\left\|f-f_{m}\right\| \leq \varepsilon \quad \text { for all } m>p_{0} .
$$

Using (2.1), it is easy to verify by induction that

$$
K^{\alpha}(f, 3 \varepsilon) \subseteq K^{\alpha}\left(f_{p_{0}+1}, \varepsilon\right)
$$


for all $\alpha<\omega_{1}$. In particular,

$$
K^{\beta_{0}}(f, 3 \varepsilon) \subseteq K^{\beta_{0}}\left(f_{p_{0}+1}, \varepsilon\right)=\emptyset .
$$

Hence $\beta(f, 3 \varepsilon) \leq \beta_{0}=\beta_{0} \cdot \gamma_{0}$.

Suppose the assertion is true for some $\gamma_{0}$. Let $\left(f_{n}\right)$ be a sequence in $\mathfrak{B}_{1}(K)$ that converges pointwise to a function $f$. Suppose there exists $\varepsilon>0$ such that $\beta\left(f_{n}, \varepsilon\right) \leq \beta_{0}$ for all $n \in \mathbb{N}$ and $\gamma\left(\left(f_{n}\right), \varepsilon\right) \leq \gamma_{0}+1$. We need to show $\beta(f, 3 \varepsilon) \leq \beta_{0} \cdot\left(\gamma_{0}+1\right)$. Since $\gamma\left(\left(f_{n}\right), \varepsilon\right) \leq \gamma_{0}+1$, we have $K^{\gamma_{0}+1}\left(\left(f_{n}\right), \varepsilon\right)=\emptyset$. For each $m \in \mathbb{N}$, let $U_{m}$ denote the $1 / m$-neighborhood of $K^{\gamma_{0}}\left(\left(f_{n}\right), \varepsilon\right)$. Denote $K \backslash U_{m}$ by $\widetilde{K}_{m}$. From Lemma 2.1(a), (b), for each $n \in \mathbb{N}, \beta_{\widetilde{K}_{m}}\left(f_{n}, \varepsilon\right) \leq \beta_{0}$ and $\gamma_{\widetilde{K}_{m}}\left(\left(f_{n}\right), \varepsilon\right) \leq \gamma_{0}$. By the inductive hypothesis, we see that

$$
\beta_{\widetilde{K}_{m}}(f, 3 \varepsilon) \leq \beta_{0} \cdot \gamma_{0}
$$

From this and applying Lemma $2.1(\mathrm{c})$ with $U=K \backslash \bar{U}_{m}, L=\widetilde{K}_{m}$ for all $m \in \mathbb{N}$, we see that $K^{\beta_{0} \cdot \gamma_{0}}(f, 3 \varepsilon) \subseteq K^{\gamma_{0}}\left(\left(f_{n}\right), \varepsilon\right)$. Let

$$
\widetilde{K}=K^{\beta_{0} \cdot \gamma_{0}}(f, 3 \varepsilon) \subseteq K^{\gamma_{0}}\left(\left(f_{n}\right), \varepsilon\right) .
$$

Then $\beta_{\widetilde{K}}\left(f_{n}, \varepsilon\right) \leq \beta_{0}$ and $\gamma_{\widetilde{K}}\left(\left(f_{n}\right), \varepsilon\right)=1$. Thus $\beta_{\widetilde{K}}(f, 3 \varepsilon) \leq \beta_{0}$ by the case when $\gamma_{0}=1$. Therefore

$$
K^{\beta_{0} \cdot\left(\gamma_{0}+1\right)}(f, 3 \varepsilon)=K^{\beta_{0} \cdot \gamma_{0}+\beta_{0}}(f, 3 \varepsilon)=\widetilde{K}^{\beta_{0}}(f, 3 \varepsilon)=\emptyset .
$$

Hence

$$
\beta(f, 3 \varepsilon) \leq \beta_{0} \cdot\left(\gamma_{0}+1\right) .
$$

Suppose $\gamma_{0}<\omega_{1}$ is a limit ordinal and the statement holds for all ordinals $\gamma<\gamma_{0}$. Let $\left(f_{n}\right) \subseteq \mathfrak{B}_{1}(K)$ be a sequence that converges pointwise to a function $f$ and let $\varepsilon>0$ be given. Suppose that $\beta\left(f_{n}, \varepsilon\right) \leq \beta_{0}$ for all $n \in \mathbb{N}$, and $\gamma\left(\left(f_{n}\right), \varepsilon\right) \leq \gamma_{0}$. Then $\gamma\left(\left(f_{n}\right), \varepsilon\right)<\gamma_{0}$ since $\gamma\left(\left(f_{n}\right), \varepsilon\right)$ must be a successor ordinal. Hence $\beta(f, 3 \varepsilon) \leq \beta_{0} \cdot \gamma\left(\left(f_{n}\right), \varepsilon\right)<\beta_{0} \cdot \gamma_{0}$.

THEOREM 2.3. Let $\left(f_{n}\right)$ be a sequence in $\mathfrak{B}_{1}(K)$ converging pointwise to a function $f$. Suppose $\sup \left\{\beta\left(f_{n}\right): n \in \mathbb{N}\right\} \leq \beta_{0}$ and $\gamma\left(\left(f_{n}\right)\right) \leq \gamma_{0}$. Then $f$ is Baire-1 and $\beta(f) \leq \beta_{0} \cdot \gamma_{0}$.

For the next corollary, recall that $\operatorname{DBSC}(K)$ is the space of all differences of bounded semicontinuous functions on $K$. It is known that $\mathcal{B}_{1}^{1}(K)$ is the closure of $\operatorname{DBSC}(K)$ in the topology of uniform convergence $([3$, Theorem 3.1]).

Corollary 2.4 ([4, Corollary 9]). Let $f \in \mathcal{B}_{1}(K)$ be the pointwise limit of a sequence $\left(f_{n}\right) \subseteq \operatorname{DBSC}(K)$. If $\gamma\left(\left(f_{n}\right)\right) \leq \omega^{\xi}$ and $\omega \leq \xi<\omega_{1}$, then $\beta(f) \leq \omega^{\xi}$. 
3. Extension of Baire-1 functions. In this section, we establish several results regarding the extension of Baire- 1 functions. They are analogs of the Tietze Extension Theorem for continuous functions. These results are applied in the next section in proving the converse of Theorem 2.3.

LemMA 3.1. Suppose that $F$ is a closed subspace of $K$ and that $f$ is a Baire-1 function on $F$. For any $\varepsilon>0$, there exists a continuous function $g: K \backslash F^{1}(f, \varepsilon) \rightarrow \mathbb{R}$ such that

$$
\|g-f\|_{F \backslash F^{1}(f, \varepsilon)} \leq \varepsilon .
$$

Proof. For any $x \in F \backslash F^{1}(f, \varepsilon)$, choose an open neighborhood $U_{x}$ of $x$ in $K$ such that $U_{x} \cap F^{1}(f, \varepsilon)=\emptyset$ and $\left|f\left(x_{1}\right)-f\left(x_{2}\right)\right|<\varepsilon$ for all $x_{1}, x_{2} \in U_{x} \cap F$. The collection $\mathcal{U}=\left\{U_{x}: x \in F \backslash F^{1}(f, \varepsilon)\right\} \cup\{K \backslash F\}$ is an open cover of $K \backslash F^{1}(f, \varepsilon)$. By [1, Theorems IX.5.3 and VIII.4.2], there exists a partition of unity $\left(\varphi_{U}\right)_{U \in \mathcal{U}}$ subordinate to $\mathcal{U}$. If $U=U_{x} \in \mathcal{U}$ for some $x \in F \backslash F^{1}(f, \varepsilon)$, let $a_{U}=f(x)$; if $U=K \backslash F$, let $a_{U}=0$. Define $g: K \backslash F^{1}(f, \varepsilon) \rightarrow \mathbb{R}$ by $g=\sum_{U \in \mathcal{U}} a_{U} \varphi_{U}$. The sum is well defined and continuous since $\left\{\operatorname{supp} \varphi_{U}\right.$ : $U \in \mathcal{U}\}$ is locally finite. Let $x \in F \backslash F^{1}(f, \varepsilon)$. Then $\mathcal{V}=\left\{U \in \mathcal{U}: \varphi_{U}(x) \neq 0\right\}$ is a finite set, $\varphi_{U}(x)>0$ for all $U \in \mathcal{V}$ and $\sum_{U \in \mathcal{V}} \varphi_{U}(x)=1$. If $U \in \mathcal{V}$, then $x \in U \cap F$; hence $U \neq K \backslash F$. Therefore, $U=U_{y}$ for some $y \in F \backslash F^{1}(f, \varepsilon)$. But then $x, y \in U_{y} \cap F$ implies that $\left|a_{U}-f(x)\right|=|f(y)-f(x)|<\varepsilon$. It follows that

$$
\begin{aligned}
|g(x)-f(x)| & =\left|\sum_{U \in \mathcal{U}} a_{U} \varphi_{U}(x)-f(x)\right|=\left|\sum_{U \in \mathcal{V}} a_{U} \varphi_{U}(x)-\sum_{U \in \mathcal{V}} f(x) \varphi_{U}(x)\right| \\
& \leq \sum_{U \in \mathcal{V}}\left|a_{U}-f(x)\right| \varphi_{U}(x)<\varepsilon .
\end{aligned}
$$

This shows that $\|g-f\|_{F \backslash F^{1}(f, \varepsilon)} \leq \varepsilon$.

THEOREM 3.2. Suppose that $F$ is a closed subspace of $K$ and that $f$ is a Baire-1 function on $F$. For any $1 \leq \beta_{0}<\omega_{1}$ and any $\varepsilon>0$, there exists $g: K \backslash F^{\beta_{0}}(f, \varepsilon) \rightarrow \mathbb{R}$ such that

$$
\|g-f\|_{F \backslash F^{\beta_{0}(f, \varepsilon)}} \leq \varepsilon
$$

and

$$
\beta_{H}(g) \leq \beta_{0} \quad \text { for all compact subsets } H \text { of } K \backslash F^{\beta_{0}}(f, \varepsilon) .
$$

Proof. Let $h: K \backslash F^{1}(f, \varepsilon) \rightarrow \mathbb{R}$ be the function obtained from Lemma 3.1. If $1 \leq \alpha<\beta_{0}$, let $\widetilde{K}=\widetilde{F}=F^{\alpha}(f, \varepsilon)$. Applying Lemma 3.1 with $\widetilde{K}, \widetilde{F}$, and the function $f$ yields a continuous function $g_{\alpha}: F^{\alpha}(f, \varepsilon) \backslash F^{\alpha+1}(f, \varepsilon) \rightarrow \mathbb{R}$ such that

$$
\left\|g_{\alpha}-f\right\|_{F^{\alpha}(f, \varepsilon) \backslash F^{\alpha+1}(f, \varepsilon)} \leq \varepsilon .
$$

Let $g=h \cup \bigcup_{\alpha<\beta_{0}} g_{\alpha}: K \backslash F^{\beta_{0}}(f, \varepsilon) \rightarrow \mathbb{R}$. Then $\|g-f\|_{F \backslash F^{\beta_{0}(f, \varepsilon)}} \leq \varepsilon$. 
Suppose that $\delta>0$ and $H$ is a compact subset of $K \backslash F^{\beta_{0}}(f, \varepsilon)$. We claim that

$$
H^{\alpha}(g, \delta) \subseteq H \cap F^{\alpha}(f, \varepsilon) \quad \text { if } 1 \leq \alpha \leq \beta_{0}
$$

This claim will be verified by induction on $\alpha$, the only nontrivial case being that for successor ordinals. Thus suppose that $\alpha$ is a successor ordinal $\leq \beta_{0}$ and either $\alpha=1$ or the claim holds for its immediate predecessor $\alpha-1$. Let $x \in H^{\alpha}(g, \delta)$. If $x \notin F^{\alpha}(f, \varepsilon)$, then there exists an open neighborhood $U$ of $x$ such that

$$
\bar{U} \cap F^{\alpha}(f, \varepsilon)=\emptyset .
$$

If $\alpha \neq 1$, then $H^{\alpha-1}(g, \delta) \subseteq F^{\alpha-1}(f, \varepsilon)$ by the inductive hypothesis. Hence

$$
\bar{U} \cap H^{\alpha-1}(g, \delta) \subseteq F^{\alpha-1}(f, \varepsilon) \backslash F^{\alpha}(f, \varepsilon) .
$$

In particular, $g=g_{\alpha-1}$ on $\bar{U} \cap H^{\alpha-1}(g, \delta)$ and hence is continuous on the same set. Similarly, if $\alpha=1$, then $g=h$ is continuous on $\bar{U} \cap H \subseteq K \backslash$ $F^{1}(f, \varepsilon)$. By Lemma 2.1(c),

$$
H^{\alpha}(g, \delta) \cap U=\left(H^{\alpha-1}(g, \delta)\right)^{1}(g, \delta) \cap U \subseteq\left(H^{\alpha-1}(g, \delta) \cap \bar{U}\right)^{1}(g, \delta)=\emptyset .
$$

Thus, $x \notin H^{\alpha}(g, \delta)$. This shows that $H^{\alpha}(g, \delta) \subseteq H \cap F^{\alpha}(f, \varepsilon)$. It follows from the claim that $H^{\beta_{0}}(g, \delta)=\emptyset$. Hence $\beta_{H}(g) \leq \beta_{0}$, as required.

We obtain the following corollaries by taking $F=K$ and $\beta_{0}=\beta_{F}(f)$ respectively.

Corollary 3.3. Let $f$ be a Baire-1 function on $K$ such that $\beta(f, \varepsilon)$ $\leq \beta_{0}$ for some $1 \leq \beta_{0}<\omega_{1}$ and $\varepsilon>0$. Then there exists $g: K \rightarrow \mathbb{R}$ such that

$$
\|g-f\| \leq \varepsilon, \quad \beta(g) \leq \beta_{0} .
$$

Corollary 3.4. Let $F$ be a closed subspace of $K$. If $f$ is a Baire-1 function on $F$, then for every $\varepsilon>0$ there exists a Baire-1 function $g$ on $K$ such that

$$
\|g-f\|_{F} \leq \varepsilon, \quad \beta_{K}(g) \leq \beta_{F}(f) .
$$

Next we show that Corollary 3.4 can be improved to an exact extension theorem (i.e., the case $\varepsilon=0$ ). In the statement of Lemma 3.5, the vacuous $\operatorname{sum} \sum_{j=1}^{0} g_{j}$ is taken to be the zero function.

Lemma 3.5. Let $F$ be a closed subspace of $K$ and let $f$ be a Baire-1 function on $F$. Then there exists a sequence $\left(g_{n}\right)$ of Baire-1 functions on $K$ such that

(a) $g_{n}$ is continuous on $K \backslash F^{1}\left(f-\sum_{j=1}^{n-1} g_{j}, 1 / 2^{n-1}\right)$ for all $n \in \mathbb{N}$,

(b) $\left\|f-\sum_{j=1}^{n} g_{j}\right\|_{F \backslash F^{1}\left(f, 1 / 4^{n-1}\right)} \leq 1 / 2^{n-1}, n \in \mathbb{N}$,

(c) $\left\|g_{n}\right\|_{K} \leq 1 / 2^{n-2}$ if $n \geq 2$, and

(d) $F^{1}\left(f-\sum_{j=1}^{n} g_{j}, \delta\right) \subseteq F^{1}\left(f, \delta / 2^{n}\right)$ if $0<\delta \leq 1 / 2^{n-2}, n \in \mathbb{N}$. 
Proof. The functions $\left(g_{n}\right)$ are constructed inductively. By Lemma 3.1, there exists a continuous function $g_{1}: K \backslash F^{1}(f, 1) \rightarrow \mathbb{R}$ such that $\left\|f-g_{1}\right\|_{F \backslash F^{1}(f, 1)} \leq 1$. Extend $g_{1}$ to a function on $K$ by defining it to be 0 on $F^{1}(f, 1)$. Then (a) and (b) hold. Condition (c) holds vacuously. Moreover, if $x \in F \backslash F^{1}(f, \delta / 2), 0<\delta \leq 2$, then there exists a neighborhood $U_{1}$ of $x$ in $F$ such that $\left|f\left(x_{1}\right)-f\left(x_{2}\right)\right|<\delta / 2$ for all $x_{1}, x_{2} \in U_{1}$. Note that since $x \in F \backslash F^{1}(f, \delta / 2), g_{1}$ is continuous at $x$. Hence there exists a neighborhood $U_{2}$ of $x$ in $F$ such that $\left|g_{1}\left(x_{1}\right)-g_{1}\left(x_{2}\right)\right|<\delta / 2$ for all $x_{1}, x_{2} \in U_{2}$. Let $U=U_{1} \cap U_{2}$. Then $U$ is a neighborhood of $x$ in $F$. For all $x_{1}, x_{2} \in U$,

$$
\left|\left(f-g_{1}\right)\left(x_{1}\right)-\left(f-g_{1}\right)\left(x_{2}\right)\right|<\delta .
$$

Hence $x \notin F^{1}\left(f-g_{1}, \delta\right)$. This proves (d).

Suppose that $g_{1}, \ldots, g_{n}$ have been chosen. By Lemma 3.1, there exists a continuous function $h: K \backslash F^{1}\left(f-\sum_{j=1}^{n} g_{j}, 1 / 2^{n}\right) \rightarrow \mathbb{R}$ such that

$$
\left\|f-\sum_{j=1}^{n} g_{j}-h\right\|_{F \backslash F^{1}\left(f-\sum_{j=1}^{n} g_{j}, 1 / 2^{n}\right)} \leq \frac{1}{2^{n}} .
$$

Define $\widetilde{h}$ on $K \backslash F^{1}\left(f-\sum_{j=1}^{n} g_{j}, 1 / 2^{n}\right)$ by $\widetilde{h}=\left(h \wedge 1 / 2^{n-1}\right) \vee\left(-1 / 2^{n-1}\right)$. Then $\widetilde{h}$ is continuous. By $(\mathrm{d}), F^{1}\left(f-\sum_{j=1}^{n} g_{j}, 1 / 2^{n}\right) \subseteq F^{1}\left(f, 1 / 4^{n}\right)$. Hence $\widetilde{h}$ is defined and continuous on $K \backslash F^{1}\left(f, 1 / 4^{n}\right)$. Moreover, it follows from (b) that

$$
\left\|f-\sum_{j=1}^{n} g_{j}\right\|_{F \backslash F^{1}\left(f, 1 / 4^{n}\right)} \leq \frac{1}{2^{n-1}} .
$$

From inequality (3.1) and the definition of $\widetilde{h}$, we have

$$
\left\|f-\sum_{j=1}^{n} g_{j}-\widetilde{h}\right\|_{F \backslash F^{1}\left(f, 1 / 4^{n}\right)} \leq\left\|f-\sum_{j=1}^{n} g_{j}-h\right\|_{F \backslash F^{1}\left(f, 1 / 4^{n}\right)} .
$$

Therefore, $\left\|f-\sum_{j=1}^{n} g_{j}-\widetilde{h}\right\|_{F \backslash F^{1}\left(f, 1 / 4^{n}\right)} \leq 1 / 2^{n}$. Now define

$$
g_{n+1}= \begin{cases}\widetilde{h} & \text { on } K \backslash F^{1}\left(f-\sum_{j=1}^{n} g_{j}, 1 / 2^{n}\right), \\ 0 & \text { otherwise. }\end{cases}
$$

Then $g_{n+1}$ is continuous on $K \backslash F^{1}\left(f-\sum_{j=1}^{n} g_{j}, 1 / 2^{n}\right)$. This proves (a). Furthermore,

$$
\left\|f-\sum_{j=1}^{n+1} g_{j}\right\|_{F \backslash F^{1}\left(f, 1 / 4^{n}\right)}=\left\|f-\sum_{j=1}^{n} g_{j}-\widetilde{h}\right\|_{F \backslash F^{1}\left(f, 1 / 4^{n}\right)} \leq \frac{1}{2^{n}} .
$$

This proves (b). Also,

$$
\left\|g_{n+1}\right\|_{K} \leq\|\widetilde{h}\|_{K \backslash F^{1}\left(f-\sum_{j=1}^{n} g_{j}, 1 / 2^{n}\right)} \leq \frac{1}{2^{n-1}}
$$


by the definition of $\widetilde{h}$. This proves (c). Finally, suppose $0<\delta \leq 1 / 2^{n-1}$. Assume that $x \in F \backslash F^{1}\left(f, \delta / 2^{n+1}\right)$. Then $x \notin F^{1}\left(f-\sum_{j=1}^{n} g_{j}, \delta / 2\right)$. Thus there exists a neighborhood $U_{1}$ of $x$ in $F$ such that

$$
\left|\left(f-\sum_{j=1}^{n} g_{j}\right)\left(x_{1}\right)-\left(f-\sum_{j=1}^{n} g_{j}\right)\left(x_{2}\right)\right|<\frac{\delta}{2}
$$

whenever $x_{1}, x_{2} \in U_{1}$. Note that since $x \in F \backslash F^{1}\left(f-\sum_{j=1}^{n} g_{j}, \delta / 2\right), g_{n+1}$ is continuous at $x$. Therefore, there exists a neighborhood $U_{2}$ of $x$ in $F$ such that $\left|g_{n+1}\left(x_{1}\right)-g_{n+1}\left(x_{2}\right)\right|<\delta / 2$ for all $x_{1}, x_{2} \in U_{2}$. Let $U=U_{1} \cap U_{2}$. Then $U$ is a neighborhood of $x$ in $F$ such that

$$
\left|\left(f-\sum_{j=1}^{n+1} g_{j}\right)\left(x_{1}\right)-\left(f-\sum_{j=1}^{n+1} g_{j}\right)\left(x_{2}\right)\right|<\delta
$$

whenever $x_{1}, x_{2} \in U$. Hence $x \notin F^{1}\left(f-\sum_{j=1}^{n+1} g_{j}, \delta\right)$. This proves (d).

TheOREM 3.6. Let $F$ be a closed subspace of $K$ and let $f$ be a Baire-1 function on $F$. Then there exists a Baire-1 function $g$ on $K$ such that

$$
g_{\mid F}=f, \quad \beta(g)=\beta_{F}(f) .
$$

Proof. Let $\left(g_{n}\right)$ be the sequence given by Lemma 3.5. Define $g$ on $K$ by

$$
g= \begin{cases}\sum_{j=1}^{\infty} g_{j} & \text { on } K \backslash F, \\ f & \text { on } F .\end{cases}
$$

Note that by Lemma $3.5(\mathrm{c}), \sum_{j=1}^{\infty} g_{j}$ converges uniformly on $K$. Hence $g$ is well defined. Obviously, $g_{\mid F}=f$.

CLAim. $K^{1}\left(g, 1 / 2^{n-3}\right) \subseteq F^{1}\left(f, 1 / 4^{n}\right)$ for all $n \in \mathbb{N}$.

Proof. Let $x \in K \backslash F^{1}\left(f, 1 / 4^{n}\right)$. We consider two cases. Suppose $x \notin F$. By Lemma 3.5(a), $g_{j}$ is continuous on $K \backslash F$ for all $j$. Since $\sum_{j=1}^{\infty} g_{j}$ converges uniformly to $g$ on $K \backslash F$, and $K \backslash F$ is an open subset of $K$, it follows that $g$ is continuous at $x$. Hence $x \notin K^{1}\left(g, 1 / 2^{n-3}\right)$. Now suppose $x \in F$. Then $x \in F \backslash F^{1}\left(f, 1 / 4^{n}\right)$. There is a neighborhood $U_{1}$ of $x$ in $K$ such that $\left|f(x)-f\left(x^{\prime}\right)\right|<1 / 4^{n}$ for all $x^{\prime} \in U_{1} \cap F$. Also, for $1 \leq k \leq n$,

$$
\begin{aligned}
F^{1}\left(f-\sum_{j=1}^{k} g_{j}, 1 / 2^{k}\right) & \subseteq F^{1}\left(f, 1 / 4^{k}\right) \quad \text { by Lemma } 3.5(\mathrm{~d}) \\
& \subseteq F^{1}\left(f, 1 / 4^{n}\right) .
\end{aligned}
$$

Since $g_{k+1}$ is continuous on $K \backslash F^{1}\left(f-\sum_{j=1}^{k} g_{j}, 1 / 2^{k}\right)$, it follows that $g_{k+1}$ is continuous on $K \backslash F^{1}\left(f, 1 / 4^{n}\right)$ for all $k, 1 \leq k \leq n$. Similarly, $F^{1}(f, 1) \subseteq$ $F^{1}\left(f, 1 / 4^{n}\right)$ and $g_{1}$ is continuous on $K \backslash F^{1}(f, 1)$ by Lemma 3.5(a); thus, $g_{1}$ is continuous on $K \backslash F^{1}\left(f, 1 / 4^{n}\right)$. Hence there exists a neighborhood $U_{2}$ of 
$x$ in $K$ such that $U_{2} \subseteq K \backslash F^{1}\left(f, 1 / 4^{n}\right)$ and

$$
\left|\sum_{j=1}^{n+1} g_{j}\left(x^{\prime}\right)-\sum_{j=1}^{n+1} g_{j}(x)\right|<1 / 2^{n} \quad \text { for all } x^{\prime} \in U_{2} .
$$

Let $U=U_{1} \cap U_{2}$. Then $U$ is a neighborhood of $x$ in $K$. If $x^{\prime} \in U \cap F$, then $x^{\prime} \in U_{1} \cap F$. Thus $\left|g\left(x^{\prime}\right)-g(x)\right|=\left|f\left(x^{\prime}\right)-f(x)\right|<1 / 4^{n}<1 / 2^{n-2}$. If $x^{\prime} \in U \backslash F$, then

$$
\begin{aligned}
\left|g\left(x^{\prime}\right)-g(x)\right| & =\left|\sum_{j=1}^{\infty} g_{j}\left(x^{\prime}\right)-f(x)\right| \\
& \leq\left|\sum_{j=1}^{n+1} g_{j}\left(x^{\prime}\right)-\sum_{j=1}^{n+1} g_{j}(x)\right|+\left|\sum_{j=1}^{n+1} g_{j}(x)-f(x)\right|+\left|\sum_{j=n+2}^{\infty} g_{j}\left(x^{\prime}\right)\right| \\
& <\frac{1}{2^{n}}+\left|\sum_{j=1}^{n+1} g_{j}(x)-f(x)\right|+\sum_{j=n+2}^{\infty}\left\|g_{j}\right\| \quad \text { since } x^{\prime} \in U_{2} \\
& \leq \frac{1}{2^{n}}+\left|\sum_{j=1}^{n+1} g_{j}(x)-f(x)\right|+\sum_{j=n+2}^{\infty} \frac{1}{2^{j-2}} \quad \text { by Lemma } 3.5(\mathrm{c}) \\
& \leq \frac{1}{2^{n}}+\frac{1}{2^{n}}+\frac{1}{2^{n-1}} \quad \text { by Lemma } 3.5(\mathrm{~b}) \text { since } x \in F \backslash F^{1}\left(f, 1 / 4^{n}\right) \\
& =\frac{1}{2^{n-2}} .
\end{aligned}
$$

Thus $\left|g\left(x^{\prime}\right)-g(x)\right|<1 / 2^{n-2}$ if $x^{\prime} \in U$. Hence $\left|g\left(x_{1}\right)-g\left(x_{2}\right)\right|<1 / 2^{n-3}$ whenever $x_{1}, x_{2} \in U$. Therefore $x \notin K^{1}\left(g, 1 / 2^{n-3}\right)$. This proves the Claim.

It follows by induction that

$$
K^{\alpha}\left(g, 1 / 2^{n-3}\right) \subseteq F^{\alpha}\left(f, 1 / 4^{n}\right) \quad \text { for } 1 \leq \alpha<\omega_{1} .
$$

Indeed, the Claim yields the assertion for $\alpha=1$. If the inclusion holds for some $\alpha, 1 \leq \alpha<\omega_{1}$, let $\widetilde{F}=F^{\alpha}\left(f, 1 / 4^{n}\right)$. Then $K^{\alpha+1}\left(g, 1 / 2^{n-3}\right) \subseteq$ $\widetilde{F}^{1}\left(g, 1 / 2^{n-3}\right)=\widetilde{F}^{1}\left(f, 1 / 2^{n-3}\right) \subseteq \widetilde{F}^{1}\left(f, 1 / 4^{n}\right)=F^{\alpha+1}\left(f, 1 / 4^{n}\right)$. Hence the inclusion holds for $\alpha+1$. If the inclusion holds for all $1 \leq \alpha^{\prime}<\alpha$, where $\alpha<\omega_{1}$ is a limit ordinal, then

$$
K^{\alpha}\left(g, 1 / 2^{n-3}\right)=\bigcap_{1 \leq \alpha^{\prime}<\alpha} K^{\alpha^{\prime}}\left(g, 1 / 2^{n-3}\right) \subseteq \bigcap_{1 \leq \alpha^{\prime}<\alpha} F^{\alpha^{\prime}}\left(f, 1 / 4^{n}\right)=F^{\alpha}\left(f, 1 / 4^{n}\right) .
$$

This proves the inclusion for $1 \leq \alpha<\omega_{1}$.

In particular, if $\beta_{F}(f)=\beta_{0}$, then $K^{\beta_{0}}\left(g, 1 / 2^{n-3}\right) \subseteq F^{\beta_{0}}\left(f, 1 / 4^{n}\right)=\emptyset$. Thus $\beta_{K}\left(g, 1 / 2^{n-3}\right) \leq \beta_{0}$ for all $n \in \mathbb{N}$. Hence $\beta_{K}(g) \leq \beta_{0}$. Of course, since $g_{\mid F}=f$, we have $\beta_{K}(g) \geq \beta_{F}(f) \geq \beta_{0}$. Therefore $\beta_{K}(g)=\beta_{0}=\beta_{F}(f)$. 
Remark 3.7. If $\beta_{F}(f)=1$, Theorem 3.6 is the familiar Tietze Extension Theorem. If $\beta_{F}(f)$ is transfinite, the conclusion of Theorem 3.6 can be obtained easily by defining the extension $g$ to be 0 on $K \backslash F$. However, we do not see a simple proof for finite $\beta_{F}(f)$.

4. Decomposition of Baire-1 functions. In this section, we give a proof of Theorem 1.2. The extension results in $\S 3$ are employed in the course of the proof.

TheOREm 4.1. Let $f$ be a Baire-1 function on $K, 1 \leq \beta_{0}, \gamma_{0}<\omega_{1}$ and $\varepsilon>0$. Then there exist

$$
\tilde{f}: K \backslash K^{\beta_{0} \cdot \gamma_{0}}(f, \varepsilon) \rightarrow \mathbb{R} \quad \text { and } \quad f_{n}: K \backslash K^{\beta_{0} \cdot \gamma_{0}}(f, \varepsilon) \rightarrow \mathbb{R}
$$

such that $\left(f_{n}\right)$ converges to $\tilde{f}$ pointwise, $\|\widetilde{f}-f\|_{K \backslash K^{\beta_{0} \cdot \gamma_{0}(f, \varepsilon)}} \leq \varepsilon, \beta_{H}\left(f_{n}\right)$ $\leq \beta_{0}$ and $\gamma_{H}\left(\left(f_{n}\right)\right) \leq \gamma_{0}$ for all compact subsets $H$ of $K \backslash K^{\beta_{0} \cdot \gamma_{0}}(f, \varepsilon)$.

Proof. For $\alpha \leq \gamma_{0}$, let $K_{\alpha}=K^{\beta_{0} \cdot \alpha}(f, \varepsilon)$. If $\alpha<\gamma_{0}$, it follows from Theorem 3.2 that there exists $g_{\alpha}: K_{\alpha} \backslash K_{\alpha+1} \rightarrow \mathbb{R}$ such that $\left\|g_{\alpha}-f\right\|_{K_{\alpha} \backslash K_{\alpha+1}} \leq \varepsilon$ and $\beta_{H}\left(g_{\alpha}\right) \leq \beta_{0}$ for all compact subsets $H$ of $K_{\alpha} \backslash K_{\alpha+1}$. List the ordinals in $\left[0, \gamma_{0}\right)$ in a (possibly finite) sequence $\left(\alpha_{n}\right)_{n=1}^{p}$. Here $p \in \mathbb{N}$ or $p=\infty$. For all $n$ and $\alpha$, let $U_{n}^{\alpha}$ be the $1 / n$-neighborhood of $K_{\alpha}$ in $K$ and set $F_{n}=\bigcup_{j=1}^{n \wedge p}\left(K_{\alpha_{j}} \backslash U_{n}^{\alpha_{j}+1}\right)$. Then $F_{n}$ is a closed subset of $K$. It is also easy to see that $K_{\alpha} \backslash U_{n}^{\alpha+1}$ and $K_{\alpha^{\prime}} \backslash U_{n}^{\alpha^{\prime}+1}$ are disjoint if $\alpha \neq \alpha^{\prime}$. Thus $\left(K_{\alpha_{j}} \backslash U_{n}^{\alpha_{j}+1}\right)_{j=1}^{n \wedge p}$ is a partition of $F_{n}$ into clopen (in $F_{n}$ ) subsets.

Now define $\widetilde{g}_{n}: F_{n} \rightarrow K$ to be $\bigcup_{j=1}^{n \wedge p} g_{\alpha_{j} \mid K_{\alpha_{j}} \backslash U_{n}^{\alpha_{j}+1}}$. Since $H=K_{\alpha_{j}} \backslash U_{n}^{\alpha_{j}+1}$ is a compact subset of $K_{\alpha_{j}} \backslash K_{\alpha_{j}+1}$, we have $\beta_{H}\left(g_{\alpha_{j}}\right) \leq \beta_{0}$. As the partition $\left(K_{\alpha_{j}} \backslash U_{n}^{\alpha_{j}+1}\right)_{j=1}^{n \wedge p}$ is clopen, it follows readily that $\beta_{F_{n}}\left(\widetilde{g}_{n}\right) \leq \beta_{0}$. By Theorem 3.6, there exists a function $f_{n}^{\prime}$ on $K$ such that $f_{n \mid F_{n}}^{\prime}=\widetilde{g}_{n}$ and $\beta_{K}\left(f_{n}^{\prime}\right)$ $\leq \beta_{0}$.

Finally, define $f_{n}$ to be $f_{n \mid K \backslash K_{\gamma_{0}}}^{\prime}$ and $\tilde{f}$ to be $\bigcup_{\alpha<\gamma_{0}} g_{\alpha \mid K_{\alpha} \backslash K_{\alpha+1}}$. It follows from the choice of the $g_{\alpha}$ 's that $\|f-\widetilde{f}\|_{K \backslash K_{\gamma_{0}}} \leq \varepsilon$. Since $\bigcup_{n=1}^{\infty} F_{n}=K \backslash K_{\gamma_{0}}$ and the sets $F_{n}$ are increasing, $\lim f_{n}=\tilde{f}$ pointwise on $K \backslash K_{\gamma_{0}}$. Suppose $H$ is a compact subset of $K \backslash K_{\gamma_{0}}$. Then $\beta_{H}\left(f_{n}\right) \leq \beta_{K}\left(f_{n}^{\prime}\right) \leq \beta_{0}$.

To complete the proof, we claim that

$$
H^{\gamma}\left(\left(f_{n}\right), \delta\right) \subseteq K_{\gamma}
$$

for any $\delta>0$ and any $\gamma \leq \gamma_{0}$. This is proved by induction on $\gamma$. The case $\gamma=0$ and the limit case are trivial. Now assume that the claim holds for some $\gamma<\gamma_{0}$. Let $x \in H^{\gamma}\left(\left(f_{n}\right), \delta\right) \backslash K_{\gamma+1}$. Choose $j_{1}, j_{2} \in \mathbb{N}$ such that $\alpha_{j_{1}}=\gamma$ and $d\left(x, K_{\gamma+1}\right) \geq 1 / j_{2}$, where $d$ is the metric on $K$. Denote $H^{\gamma}\left(\left(f_{n}\right), \delta\right)$ by $L$ and the $1 /\left(2 j_{0}\right)$-ball in $K$ centered at $x$ by $U$, where $j_{0}=\max \left\{j_{1}, 2 j_{2}\right\}$. Note 
that $L \subseteq K_{\gamma}$ by the inductive hypothesis. For all $n \geq j_{0}=\max \left\{j_{1}, 2 j_{2}\right\}$,

$$
L \cap U \subseteq L \cap \bar{U} \subseteq K_{\alpha_{j_{1}}} \backslash U_{n}^{\alpha_{j_{1}}+1} \subseteq F_{n} .
$$

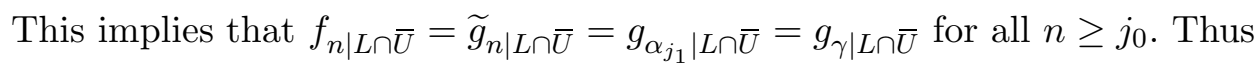
$(L \cap \bar{U})^{1}\left(\left(f_{n}\right), \delta\right)=\emptyset$. By Lemma 2.1(d),

$$
L^{1}\left(\left(f_{n}\right), \delta\right) \cap(L \cap U)=\emptyset .
$$

In particular,

$$
x \notin L^{1}\left(\left(f_{n}\right), \delta\right)=H^{\gamma+1}\left(\left(f_{n}\right), \delta\right) .
$$

Since $x \in H^{\gamma}\left(\left(f_{n}\right), \delta\right) \backslash K_{\gamma+1}$ is arbitrary, this shows that $H^{\gamma+1}\left(\left(f_{n}\right), \delta\right) \subseteq$ $K_{\gamma+1}$.

In particular, if $\beta_{K}(f) \leq \beta_{0} \cdot \gamma_{0}$, we have the following.

TheOREM 4.2. Let $f$ be a Baire-1 function on $K, 1 \leq \beta_{0}, \gamma_{0}<\omega_{1}$, and $\beta(f) \leq \beta_{0} \cdot \gamma_{0}$. For any $\varepsilon>0$, there exist $\widetilde{f}: K \rightarrow \mathbb{R}$ and a sequence of functions $f_{n}: K \rightarrow \mathbb{R}$ such that $\left(f_{n}\right)$ converges to $\tilde{f}$ pointwise, $\|\tilde{f}-f\| \leq \varepsilon$, $\beta\left(f_{n}\right) \leq \beta_{0}$ for all $n \in \mathbb{N}$, and $\gamma\left(\left(f_{n}\right)\right) \leq \gamma_{0}$.

A couple more preparatory steps will allow us to improve Theorem 4.2 to an exact result (i.e., with $\varepsilon=0$ ) when $\gamma_{0}$ is of the right form.

Theorem 4.3 ([3, Lemma 2.5]). If $\left(f_{n}\right)$ and $\left(g_{n}\right)$ are two sequences of real-valued functions on $K$ such that $\gamma\left(\left(f_{n}\right)\right) \leq \omega^{\xi}$ and $\gamma\left(\left(g_{n}\right)\right) \leq \omega^{\xi}$ for some $\xi<\omega_{1}$, then $\gamma\left(\left(f_{n}+g_{n}\right)\right) \leq \omega^{\xi}$.

Proposition 4.4. For $1 \leq \xi<\omega_{1}, \mathfrak{B}_{1}^{\xi}(K)=\left\{f \in \mathbb{R}^{K}: \beta(f) \leq \omega^{\xi}\right\}$ is a vector subspace of $\mathbb{R}^{K}$ that is closed under the topology of uniform convergence.

We postpone the proof of Proposition 4.4 until the next section. We are now ready to prove the converse of Theorem 2.3 in certain cases.

TheOREM 4.5. If $f \in \mathfrak{B}_{1}(K)$ and $\beta(f) \leq \beta_{0} \cdot \omega^{\gamma_{0}}$ for some $1 \leq \beta_{0}<\omega_{1}$ and $\gamma_{0}<\omega_{1}$, then there exists $\left(f_{n}\right) \subseteq \mathfrak{B}_{1}(K)$ such that $\left(f_{n}\right)$ converges pointwise to $f, \beta\left(f_{n}\right) \leq \beta_{0}$ for all $n \in \mathbb{N}$ and $\gamma\left(\left(f_{n}\right)\right) \leq \omega^{\gamma_{0}}$.

Proof. First we assume $\beta_{0}$ is of the form $\omega^{\alpha_{0}}$, where $\alpha_{0}<\omega_{1}$. By Theorem 4.2 there exist a sequence $\left(f_{n}^{1}\right) \subseteq \mathfrak{B}_{1}(K)$ and a function $f^{1} \in \mathfrak{B}_{1}(K)$ such that $\beta\left(f_{n}^{1}\right) \leq \omega^{\alpha_{0}}$ for all $n,\left(f_{n}^{1}\right)$ converges pointwise to $f^{1},\left\|f^{1}-f\right\|$ $\leq 1 / 2$, and $\gamma\left(\left(f_{n}^{1}\right)\right) \leq \omega^{\gamma_{0}}$. Then $\beta\left(f^{1}\right) \leq \omega^{\alpha_{0}} \cdot \omega^{\gamma_{0}}=\omega^{\alpha_{0}+\gamma_{0}}$ by Theorem 2.3. This implies that $\beta\left(f-f^{1}\right) \leq \omega^{\alpha_{0}+\gamma_{0}}$ by Proposition 4.4. Hence there exist $\left(f_{n}^{2}\right) \subseteq \mathfrak{B}_{1}(K)$ and $f^{2}$ such that $\beta\left(f_{n}^{2}\right) \leq \omega^{\alpha_{0}}$ for all $n \in \mathbb{N}$, $\left(f_{n}^{2}\right)$ converges pointwise to $f^{2},\left\|f-f^{1}-f^{2}\right\| \leq 1 / 2^{2}$, and $\gamma\left(\left(f_{n}^{2}\right)\right) \leq \omega^{\gamma_{0}}$. We may assume that $\left\|f_{n}^{2}\right\| \leq 1 / 2$ for all $n \in \mathbb{N}$, for otherwise, simply replace 
$f_{n}^{2}$ by $\widehat{f}_{n}^{2}=\left(f_{n}^{2} \vee(-1 / 2)\right) \wedge 1 / 2$. Continuing, we obtain $f^{m}$ and $\left(f_{n}^{m}\right)_{n=1}^{\infty}$ for each $m$ such that

- $\left\|f_{n}^{m}\right\| \leq 1 / 2^{m-1}, m \geq 2$,

- $\beta\left(f_{n}^{m}\right) \leq \omega^{\alpha_{0}}$ for all $m, n \in \mathbb{N}$,

- $\gamma\left(\left(f_{n}^{m}\right)_{n}\right) \leq \omega^{\gamma_{0}}$ for all $m \in \mathbb{N}$,

- $f^{m}=\lim _{n} f_{n}^{m}$ (pointwise) for all $m \in \mathbb{N}$,

- $\sum_{m=1}^{\infty} f^{m}$ converges uniformly to $f$ on $K$.

Let $g_{n}^{m}=f_{n}^{1}+f_{n}^{2}+\ldots+f_{n}^{m}$ and $g_{n}=\sum_{m=1}^{\infty} f_{n}^{m}$. By Theorem 4.3, $\gamma\left(\left(g_{n}^{m}\right)_{n}\right) \leq \omega^{\gamma_{0}}$ for all $m \in \mathbb{N}$. Given $\varepsilon>0$, there exists $m_{0}$ such that $\left\|g_{n}^{m_{0}}-g_{n}\right\| \leq \varepsilon$ for all $n \in \mathbb{N}$. Then $K^{\omega^{\gamma_{0}}}\left(\left(g_{n}\right), 3 \varepsilon\right) \subseteq K^{\omega^{\gamma_{0}}}\left(\left(g_{n}^{m_{0}}\right), \varepsilon\right)=\emptyset$. Therefore $\gamma\left(\left(g_{n}\right)\right) \leq \omega^{\gamma_{0}}$. By Proposition $4.4, \beta\left(g_{n}^{m}\right) \leq \omega^{\alpha_{0}}$ for all $m, n$. Therefore, $\beta\left(g_{n}\right) \leq \omega^{\alpha_{0}}$ by Proposition 4.4. Moreover,

$$
\begin{aligned}
\lim _{n} g_{n} & =\lim _{n} \lim _{m} g_{n}^{m}=\lim _{m} \lim _{n} g_{n}^{m} \\
& =\lim _{m} \sum_{k=1}^{m} f^{k}=f \quad \text { pointwise. }
\end{aligned}
$$

This proves the theorem in case $\beta_{0}=\omega^{\alpha_{0}}$, with $\left(g_{n}\right)$ in place of $\left(f_{n}\right)$.

For a general nonzero countable ordinal $\beta_{0}$, write $\beta_{0}$ in Cantor normal form as

$$
\beta_{0}=\omega^{\beta_{1}} \cdot m_{1}+\omega^{\beta_{2}} \cdot m_{2}+\ldots+\omega^{\beta_{k}} \cdot m_{k},
$$

where $k, m_{1}, \ldots, m_{k} \in \mathbb{N}, \omega_{1}>\beta_{1}>\ldots>\beta_{k}$. If $\gamma_{0} \neq 0$, then $\beta_{0} \cdot \omega^{\gamma_{0}}=$ $\omega^{\beta_{1}} \cdot \omega^{\gamma_{0}}$. By the previous case, there exists $\left(f_{n}\right) \subseteq \mathfrak{B}_{1}(K)$ such that $\beta\left(f_{n}\right) \leq$ $\omega^{\beta_{1}} \leq \beta_{0}, \gamma\left(\left(f_{n}\right)\right) \leq \omega^{\gamma_{0}}$ and $\left(f_{n}\right)$ converges pointwise to $f$. If $\gamma_{0}=0$, take $f_{n}=f$ for all $n$. Then $\beta\left(f_{n}\right) \leq \beta_{0}$ for all $n, \gamma\left(\left(f_{n}\right)\right)=1=\omega^{\gamma_{0}}$ and $\left(f_{n}\right)$ converges pointwise to $f$.

The combination of Theorem 2.3 and Corollary 4.6 yields Theorem 1.2. It is clear from the proof of Theorem 4.5 that the sequence $\left(f_{n}\right)$ in the statement of Theorem 4.5 may be chosen to be bounded if $f$ is bounded.

Corollary 4.6. Let $f \in \mathfrak{B}_{1}^{\xi}(K)$, respectively, $\mathcal{B}_{1}^{\xi}(K)$, for some $\xi<\omega_{1}$. For all countable ordinals $\mu, \nu$ such that $\mu+\nu \geq \xi$, there exists a sequence $\left(f_{n}\right) \subseteq \mathfrak{B}_{1}^{\mu}(K)$, respectively, a bounded sequence $\left(f_{n}\right) \subseteq \mathcal{B}_{1}^{\mu}(K)$, such that $f_{n} \rightarrow f$ pointwise and $\gamma\left(\left(f_{n}\right)\right) \leq \omega^{\nu}$.

We do not know if Theorem 4.5 holds without the restriction on the form of the ordinal $\gamma\left(\left(f_{n}\right)\right)$.

Problem 4.7. Is it true that if $f \in \mathfrak{B}_{1}(K)$ with $\beta(f) \leq \beta_{0} \cdot \gamma_{0}$ for some countable ordinals $\beta_{0}$ and $\gamma_{0}$, then there exists a sequence $\left(f_{n}\right)$ converging pointwise to $f$ so that $\sup _{n} \beta\left(f_{n}\right) \leq \beta_{0}$ and $\gamma\left(\left(f_{n}\right)\right) \leq \gamma_{0}$ ?

As another application of our results, we give the proof of another characterization of the classes $\mathcal{B}_{1}^{\xi}(K)$ due to Kechris and Louveau. 
Definition 4.8 ([3, Section 4$])$. A family $\left\{\Phi_{\xi}: 0 \leq \xi<\omega_{1}\right\}$ of realvalued functions on $K$ is defined as follows:

$$
\begin{aligned}
& \Phi_{0}=C(K), \\
& \Phi_{\xi+1}=\{f: f \text { is the pointwise limit of a bounded sequence } \\
&\left.\left(f_{n}\right) \subseteq \Phi_{\xi} \text { such that } \gamma\left(\left(f_{n}\right)\right) \leq \omega\right\},
\end{aligned}
$$

and for limit ordinals $\lambda$,

$$
\Phi_{\lambda}=\left\{f: f \text { is the uniform limit of a bounded sequence }\left(f_{n}\right) \subseteq \bigcup_{\xi<\lambda} \Phi_{\xi}\right\} \text {. }
$$

Corollary 4.9 ([3, Theorem 4.2]). For each $\xi<\omega_{1}, \mathcal{B}_{1}^{\xi}(K)=\Phi_{\xi}$.

Proof. The case $\xi=0$ is trivial. Suppose the assertion holds for some $\xi<\omega_{1}$. If $f \in \mathcal{B}_{1}^{\xi+1}(K)$, it follows from Corollary 4.6 that $f$ is the pointwise limit of a bounded sequence $\left(f_{n}\right)$ in $\mathcal{B}_{1}^{\xi}(K)$ such that $\gamma\left(\left(f_{n}\right)\right) \leq \omega$. Since $\mathcal{B}_{1}^{\xi}(K)=\Phi_{\xi}$ by the inductive hypothesis, $f \in \Phi_{\xi+1}$. Conversely, if $f \in \Phi_{\xi+1}$, then $f$ is the pointwise limit of a sequence $\left(f_{n}\right)$ in $\Phi_{\xi}$ with $\gamma\left(\left(f_{n}\right)\right) \leq \omega$. Since $\Phi_{\xi}=\mathcal{B}_{1}^{\xi}(K)$, it follows that $\beta(f) \leq \omega^{\xi+1}$ by Theorem 2.3. Thus $f \in \mathcal{B}_{1}^{\xi+1}(K)$.

Now assume that the assertion holds for all $\xi^{\prime}<\xi$, where $\xi$ is a countable limit ordinal. Let $f \in \Phi_{\xi}$. By the inductive hypothesis, $\Phi_{\xi^{\prime}}=\mathcal{B}_{1}^{\xi^{\prime}}(K) \subseteq$ $\mathcal{B}_{1}^{\xi}(K)$ for $\xi^{\prime}<\xi$. Hence $f$ is the uniform limit of a sequence in $\mathcal{B}_{1}^{\xi}(K)$, and thus belongs to $\mathcal{B}_{1}^{\xi}(K)$. Conversely, assume that $f \in \mathcal{B}_{1}^{\xi}(K)$. For every $n \in \mathbb{N}$, there exists $\xi_{n}<\xi$ such that $\beta(f, 1 / n) \leq \omega^{\xi_{n}}$. By Corollary 3.3, there exists $f_{n} \in \mathcal{B}_{1}^{\xi_{n}}(K)=\Phi_{\xi_{n}}$ such that $\left\|f-f_{n}\right\| \leq 1 / n$. Thus $f \in \Phi_{\xi}$, as required.

REMARK 4.10. If a family $\left\{\Psi_{\xi}: 0 \leq \xi<\omega_{1}\right\}$ is defined in a similar way to $\left\{\Phi_{\xi}: 0 \leq \xi<\omega_{1}\right\}$ except that the boundedness condition on the sequence $\left(f_{n}\right)$ is removed, then $\Psi_{\xi}=\mathfrak{B}_{1}^{\xi}(K)$ for all $\xi<\omega_{1}$.

5. Optimal limit of continuous functions. In this section we prove the equivalence of the indices $\beta$ and $\gamma$ for functions in $\mathfrak{B}_{1}(K)$ in the same sense that was established for $\mathcal{B}_{1}(K)$ in Theorem 2.3 of [3]. Namely, it is shown that for all $f \in \mathfrak{B}_{1}(K), \beta(f)$ is the smallest ordinal $\gamma_{0}$ for which there exists a sequence $\left(f_{n}\right)$ in $C(K)$ converging pointwise to $f$ and satisfying $\gamma\left(\left(f_{n}\right)\right) \leq \gamma_{0}$. Note that this result is also the converse of Theorem 2.3 when $\beta_{0}=1$.

Definition 5.1. Let $\left(f_{n}\right) \subseteq \mathbb{R}^{K}$ and $f \in \mathbb{R}^{K}$. We write:

(a) $\left(g_{n}\right) \prec\left(f_{n}\right)$ if $\left(g_{n}\right)$ is a convex block combination of $\left(f_{n}\right)$, i.e., there exist a sequence of nonnegative real numbers $\left(a_{k}\right)$ and a strictly increasing 
sequence $\left(p_{n}\right)$ in $\mathbb{N}$ such that $\sum_{k=p_{n-1}+1}^{p_{n}} a_{k}=1$ and $g_{n}=\sum_{k=p_{n-1}+1}^{p_{n}} a_{k} f_{k}$ for all $n\left(p_{0}=0\right)$,

(b) $\left(g_{n}\right) \stackrel{a}{\prec}\left(f_{n}\right)$ if there exists $m \in \mathbb{N}$ such that $\left(g_{n}\right)_{n=m}^{\infty} \prec\left(f_{n}\right)$,

(c) $[f]_{-M}^{M}=(f \vee-M) \wedge M$, where $0 \leq M \in \mathbb{R}$.

The easy proof of the next lemma is left to the reader.

LEMMA 5.2. If $\left(g_{n}\right) \stackrel{a}{\prec}\left(f_{n}\right)$, then $\gamma\left(\left(g_{n}\right), \varepsilon\right) \leq \gamma\left(\left(f_{n}\right), \varepsilon\right)$ for all $\varepsilon>0$.

LEMma 5.3. Let $f$ be a Baire-1 function on $K$. Suppose $\mathcal{H}$ is a countable collection of compact subsets of $K$ such that $\|f\|_{H}<\infty$ for all $H \in \mathcal{H}$ and $\bigcup_{H \in \mathcal{H}} H=K$. Then there exists $\left(f_{n}\right) \subseteq C(K)$ such that

(i) $f_{n} \rightarrow f$ pointwise,

(ii) $\left(f_{n \mid H}\right)$ is a bounded subset of $C(H)$ for all $H \in \mathcal{H}$.

Proof. Write $\mathcal{H}$ as a sequence $\left(H_{m}\right)_{m=1}^{\infty}$. Without loss of generality, assume that $H_{m} \subseteq H_{m+1}$ for all $m \in \mathbb{N}$. Since $f$ is Baire- 1 , there exists $\left(f_{n}^{0}\right) \subseteq C(K)$ such that $\left(f_{n}^{0}\right)$ converges pointwise to $f$. Assume that $\left(f_{n}^{m-1}\right)_{n} \subseteq C(K)$ has been chosen so that $\lim _{n} f_{n}^{m-1}=f$ pointwise. If $m, n \in \mathbb{N}$, let $U_{n}^{m}$ be the $1 / n$-neighborhood of $H_{m}$ in $K$ and let $M_{m}=$ $\|f\|_{H_{m}}$. For all $n$, the function $\left[f_{n}^{m-1}\right]_{-M_{m} \mid H_{m}}^{M_{m}} \cup f_{n \mid K \backslash U_{n}^{m}}^{m-1}$ is continuous on $H_{m} \cup\left(K \backslash U_{n}^{m}\right)$. Let $f_{n}^{m}$ be its continuous extension onto $K$. If $x \in H_{m}$, then $\lim _{n} f_{n}^{m}(x)=\lim _{n}\left[f_{n}^{m-1}(x)\right]_{-M_{m}}^{M_{m}}=[f(x)]_{-M_{m}}^{M_{m}}=f(x)$ since $\|f\|_{H_{m}}=M_{m}$. If $x \notin H_{m}$, then there exists $n_{0}$ such that $x \in K \backslash U_{n_{0}}^{m}$; thus $x \in K \backslash U_{n}^{m}$ for all $n \geq n_{0}$. Therefore $f_{n}^{m}(x)=f_{n}^{m-1}(x)$ for all $n \geq n_{0}$. Hence $\lim _{n} f_{n}^{m}(x)$ $=f(x)$. Thus $\lim _{n} f_{n}^{m}=f$ pointwise. Now for each $n \in \mathbb{N}$, let $f_{n}=f_{n}^{n}$. Since $H_{m} \subseteq H_{n}$ for all $n \geq m$, on $H_{m}$ we have

$$
\begin{aligned}
f_{n} & =f_{n}^{n}=\left[f_{n}^{n-1}\right]_{-M_{n}}^{M_{n}} \\
& =\left[\left[f_{n}^{n-2}\right]_{-M_{n-1}}^{M_{n-1}}\right]_{-M_{n}}^{M_{n}}=\ldots=\left[\ldots\left[\left[f_{n}^{m-1}\right]_{-M_{m}}^{M_{m}}\right]_{-M_{m+1}}^{M_{m+1}} \ldots\right]_{-M_{n}}^{M_{n}} \\
& =\left[f_{n}^{m-1}\right]_{-M_{m}}^{M_{m}} \text { as } M_{m} \leq M_{m+1} \leq \ldots \leq M_{n} .
\end{aligned}
$$

Thus $f_{n}=\left[f_{n}^{m-1}\right]_{-M_{m}}^{M_{m}}$ on $H_{m}$ for all $n \geq m$. In particular, on the set $H_{m}$,

$$
\lim _{n} f_{n}=\left[\lim _{n} f_{n}^{m-1}\right]_{-M_{m}}^{M_{m}}=[f]_{-M_{m}}^{M_{m}}=f
$$

since $\|f\|_{H_{m}}=M_{m}$. As $K=\bigcup H_{m}$, we see that $f_{n} \rightarrow f$ pointwise. Also, for each $m,\left(f_{n \mid H_{m}}\right)_{n=m}^{\infty}$ is bounded (by $\left.M_{m}\right)$ in $C\left(H_{m}\right)$; thus $\left(f_{n \mid H_{m}}\right)_{n=1}^{\infty}$ is bounded in $C\left(H_{m}\right)$.

For the next lemma, recall that for a real-valued function $f$ defined on a set $S, \operatorname{osc}(f, S)=\sup \left\{\left|f\left(s_{1}\right)-f\left(s_{2}\right)\right|: s_{1}, s_{2} \in S\right\}$.

LEMMA 5.4. Let $\left(f_{n}\right)$ be bounded in $C(H)$, where $H$ is a compact metric space. Suppose $\left(f_{n}\right)$ converges pointwise to $f$ and $H^{1}(f, \varepsilon)=\emptyset$ for some $\varepsilon>0$. Then there exists $\left(g_{n}\right) \prec\left(f_{n}\right)$ such that $H^{1}\left(\left(g_{n}\right), 7 \varepsilon\right)=\emptyset$. 
Proof. By Corollary 3.3, there exists $\tilde{f} \in C(H)$ such that $\|f-\widetilde{f}\|_{H}$ $\leq \varepsilon$. Then $\left(f_{n}-\widetilde{f}\right)$ is bounded in $C(H), f_{n}-\widetilde{f} \rightarrow f-\widetilde{f}$ pointwise and $\operatorname{osc}(f-\widetilde{f}, H) \leq 2 \varepsilon$. By the first statement in the proof of Theorem 2.3 in [3], there exists $\left(h_{n}\right) \prec\left(f_{n}-\widetilde{f}\right)$ such that $\left\|h_{n}-(f-\widetilde{f})\right\|_{H} \leq 3 \varepsilon$. Let $g_{n}=h_{n}+\tilde{f}$ for all $n \in \mathbb{N}$. Then $\left(g_{n}\right) \prec\left(f_{n}\right)$ and $\left\|g_{n}-f\right\|_{H} \leq 3 \varepsilon$ for all $n \in \mathbb{N}$. It follows that $H^{1}\left(\left(g_{n}\right), 7 \varepsilon\right)=\emptyset$.

Theorem 5.5. Let $f$ be a Baire-1 function on $K$. There exists a sequence $\left(f_{n}\right) \subseteq C(K)$ such that $\left(f_{n}\right)$ converges pointwise to $f$ and $\gamma\left(\left(f_{n}\right)\right)$ $=\beta(f)$.

Proof. Let $\beta_{0}=\beta(f)$. For each $\alpha<\beta_{0}$, and all $m, j \in \mathbb{N}$, let $U_{m, j}^{\alpha}$ be the $1 / j$-neighborhood of $K^{\alpha}(f, 1 / m)$ in $K$. Define

$$
\mathcal{H}=\left\{K^{\alpha}(f, 1 / m) \backslash U_{m, j}^{\alpha+1}: \alpha<\beta_{0}, m, j \in \mathbb{N}\right\} .
$$

Then $\mathcal{H}$ is a countable collection of compact subsets of $K$ such that $\bigcup_{H \in \mathcal{H}} H$ $=K$. If $\alpha<\beta_{0}$ and $m, j \in \mathbb{N}$, by Lemma 3.1, there is a continuous function $g$ on $H=K^{\alpha}(f, 1 / m) \backslash U_{m, j}^{\alpha+1}$ such that $\|g-f\|_{H} \leq 1 / m$. Hence $\|f\|_{H}<\infty$ for all $H \in \mathcal{H}$. By Lemma 5.3, there exists $\left(g_{n}\right) \subseteq C(K)$ such that $\left(g_{n}\right)$ converges pointwise to $f$ and $\left(g_{n \mid H}\right)$ is bounded in $C(H)$ for all $H \in \mathcal{H}$.

List the elements of $\mathcal{H}$ in a sequence $\left(H_{k}\right)_{k=1}^{\infty}$. Take $\varepsilon_{k}=1 / m$ if $H_{k}$ is of the form $K^{\alpha}(f, 1 / m) \backslash U_{m, j}^{\alpha+1}$ for some $\alpha, m, j$. Let $\left(g_{n}^{0}\right)=\left(g_{n}\right)$. Suppose $\left(g_{n}^{k-1}\right)_{n} \prec\left(g_{n}\right)_{n}$ has been chosen. Then $\left(g_{n}^{k-1}\right)_{n}$ converges to $f$ pointwise, $\left(g_{n \mid H_{k}}^{k-1}\right)$ is a bounded sequence in $C\left(H_{k}\right)$, and $\left(H_{k}\right)^{1}\left(f, \varepsilon_{k}\right)=\emptyset$. By Lemma 5.4, there exists $\left(g_{n}^{k}\right)_{n} \prec\left(g_{n}^{k-1}\right)_{n}$ such that $\left(H_{k}\right)^{1}\left(\left(g_{n}^{k}\right)_{n}, 7 \varepsilon_{k}\right)=\emptyset$. Let $f_{n}=g_{n}^{n}$ for all $n \in \mathbb{N}$. Then $\left(f_{n}\right) \prec\left(g_{n}\right)$. Therefore $\left(f_{n}\right) \subseteq C(K)$ and $\left(f_{n}\right)$ converges pointwise to $f$.

We claim that for all $m \in \mathbb{N}$ and for all $\alpha \leq \beta_{0}$,

$$
K^{\alpha}\left(\left(f_{n}\right), 7 / m\right) \subseteq K^{\alpha}(f, 1 / m) .
$$

We prove the claim by induction on $\alpha$. The claim is trivial if $\alpha=0$ or $\alpha$ is a limit ordinal. Assume that $\alpha \leq \beta_{0}$ is a successor ordinal and that the claim holds for $\alpha-1$. Let $x \in K^{\alpha}\left(\left(f_{n}\right), 7 / m\right)$. Then $x \in K^{\alpha-1}\left(\left(f_{n}\right), 7 / m\right) \subseteq K^{\alpha-1}(f, 1 / m)$. If $x \notin K^{\alpha}(f, 1 / m)$, then there exists $j \in \mathbb{N}$ such that $d\left(x, K^{\alpha}(f, 1 / m)\right)>1 / j$. Choose $k$ such that $H_{k}=K^{\alpha-1}(f, 1 / m) \backslash U_{m, j}^{\alpha}$. Then $\left(f_{n}\right) \stackrel{a}{\prec}\left(g_{n}^{k}\right)_{n}$ and $\gamma_{H_{k}}\left(\left(g_{n}^{k}\right)_{n}, 7 \varepsilon_{k}\right) \leq 1$ since $\left(H_{k}\right)^{1}\left(\left(g_{n}^{k}\right)_{n}, 7 \varepsilon_{k}\right)=\emptyset$. By Lemma 5.2, $\left(H_{k}\right)^{1}\left(\left(f_{n}\right), 7 \varepsilon_{k}\right)=\emptyset$. Thus $\left(H_{k}\right)^{1}\left(\left(f_{n}\right), 7 / m\right)=\emptyset$. But since $d\left(x, K^{\alpha}(f, 1 / m)\right)>1 / j$, there exists an open set $U$ in $\widetilde{K}=K^{\alpha-1}(f, 1 / m)$ such that $x \in U \subseteq H_{k} \subseteq \widetilde{K}$. By Lemma 2.1(d), $(\widetilde{K})^{1}\left(\left(f_{n}\right), 7 / m\right) \cap U \subseteq\left(H_{k}\right)^{1}\left(\left(f_{n}\right), 7 / m\right)=\emptyset$. Therefore $x \notin(\widetilde{K})^{1}\left(\left(f_{n}\right), 7 / m\right) \supseteq K^{\alpha}\left(\left(f_{n}\right), 7 / m\right)$, a contradiction. This proves the claim. 
From the claim, $K^{\beta_{0}}\left(\left(f_{n}\right), 7 / m\right) \subseteq K^{\beta_{0}}(f, 1 / m)=\emptyset$ for all $m \in \mathbb{N}$. Therefore $\gamma\left(\left(f_{n}\right)\right) \leq \beta_{0}$. Since $\gamma\left(\left(f_{n}\right)\right) \geq \beta_{0}$ by [3, Proposition 2.1] (or Theorem 2.3), $\gamma\left(\left(f_{n}\right)\right)=\beta_{0}=\beta(f)$.

REMARK 5.6. Theorem 2.3 of [3] actually implies that if $\left(f_{n}\right)$ is a bounded sequence in $C(K)$ converging pointwise to some $f \in \mathcal{B}_{1}(K)$, then there exists $\left(g_{n}\right) \prec\left(f_{n}\right)$ such that $\gamma\left(\left(g_{n}\right)\right)=\beta(f)$. This does not hold in general for unbounded sequences of functions. Indeed, let $K=[0,1]$ and for each $n \in \mathbb{N}$ let $f_{n}$ be a continuous function that vanishes outside $[1 /(n+1), 1 / n]$ such that $\int_{K} f_{n}=1$. Then $\left(f_{n}\right)$ converges pointwise to $f=0$. If $\left(g_{n}\right) \prec\left(f_{n}\right)$, then $\int_{K} g_{n}=1$ for all $n \in \mathbb{N}$. Thus $\left(g_{n}\right)$ does not converge uniformly to $f$, i.e., $\gamma\left(\left(g_{n}\right)\right)>1=\beta(f)$.

Proof of Proposition 4.4. It is easy to see that af $\in \mathfrak{B}_{1}^{\xi}(K)$ for all $f \in$ $\mathfrak{B}_{1}^{\xi}(K)$ and $a \in \mathbb{R}$. If $f, g \in \mathfrak{B}_{1}^{\xi}(K)$, then by Theorem 5.5 there exist two sequences $\left(f_{n}\right)$ and $\left(g_{n}\right)$ of continuous functions converging pointwise to $f$ and $g$ respectively such that $\gamma\left(\left(f_{n}\right)\right) \leq \omega^{\xi}$ and $\gamma\left(\left(g_{n}\right)\right) \leq \omega^{\xi}$. According to Theorem 4.3, $\gamma\left(\left(f_{n}+g_{n}\right)\right) \leq \omega^{\xi}$. Hence by Theorem 2.3, $f+g \in \mathfrak{B}_{1}^{\xi}(K)$. Finally, given $f \in \overline{\mathfrak{B}_{1}^{\xi}(K)}$ and $\varepsilon>0$, choose $g \in \mathfrak{B}_{1}^{\xi}(K)$ such that $\|f-g\|$ $\leq \varepsilon / 3$. Then $K^{\omega^{\xi}}(f, \varepsilon) \subseteq K^{\omega^{\xi}}(g, \varepsilon / 3)=\emptyset$. Thus $f \in \mathfrak{B}_{1}^{\xi}(K)$.

6. Product of Baire-1 functions. In [3], it is observed that the classes $\mathcal{B}_{1}^{\xi}(K), \xi<\omega_{1}$, are closed under multiplication. However, it is relatively easy to see that this fails for the classes $\mathfrak{B}_{1}^{\xi}(K)$. In this section, we show that if $f \in$ $\mathfrak{B}_{1}^{\xi_{1}}(K)$ and $g \in \mathfrak{B}_{1}^{\xi_{2}}(K)$, then $f g \in \mathfrak{B}_{1}^{\xi}(K)$, where $\xi=\max \left\{\xi_{1}+\xi_{2}, \xi_{2}+\xi_{1}\right\}$. It is also shown that the result is sharp. The proof of the next lemma is left to the reader.

Lemma 6.1. If $f$ is bounded and $\gamma\left(\left(g_{n}\right)\right) \leq \xi$, then $\gamma\left(\left(f g_{n}\right)\right) \leq \xi$.

LEMma 6.2. If $f \in \mathcal{B}_{1}^{\xi_{1}}(K)$ and $g \in \mathfrak{B}_{1}^{\xi_{2}}(K)$, then $f g \in \mathfrak{B}_{1}^{\xi_{1}}+\xi_{2}(K)$.

Proof. By Theorem 5.5, there exists a sequence $\left(g_{n}\right) \subseteq C(K)$ converging to $g$ pointwise such that $\gamma\left(\left(g_{n}\right)\right) \leq \omega^{\xi_{2}}$. For each $n \in \mathbb{N}, g_{n} \in C(K)$ $\subseteq \mathcal{B}_{1}^{\xi_{1}}(K)$ and $f \in \mathcal{B}_{1}^{\xi_{1}}(K)$. By [3] (see the remark in [3, p. 217]), we have $f g_{n} \in \mathcal{B}_{1}^{\xi_{1}}(K)$. Lemma 6.1 implies that $\gamma\left(\left(f g_{n}\right)\right) \leq \omega^{\xi_{2}}$. Since $\left(f g_{n}\right)$ converges to $f g$ pointwise, it follows from Theorem 2.3 that $\beta(f g) \leq \omega^{\xi_{1}+\xi_{2}}$, i.e., $f g \in \mathfrak{B}_{1}^{\xi_{1}+\xi_{2}}(K)$.

Now suppose $f \in \mathfrak{B}_{1}^{\xi_{1}}(K)$ and $g \in \mathfrak{B}_{1}^{\xi_{2}}(K)$. By Lemma 3.1, for all $\alpha<\omega^{\xi_{2}}$, there is a continuous function $g_{\alpha}: K^{\alpha}(g, 1) \backslash K^{\alpha+1}(g, 1) \rightarrow \mathbb{R}$ such that

$$
\left\|g_{\alpha}-g\right\|_{K^{\alpha}(g, 1) \backslash K^{\alpha+1}(g, 1)} \leq 1 .
$$


Let $h=\bigcup_{\alpha<\omega \xi_{2}} g_{\alpha}$. It follows from the proof of Theorem 3.2 that $\beta(h) \leq \omega^{\xi_{2}}$. Given a closed set $H \subseteq K$, we write

$$
\mathrm{d}_{f}(H)=\left\{x \in H: \limsup _{\substack{y \rightarrow x \\ y \in H}}|f(y)|=\infty\right\} .
$$

It is easy to see that $\mathrm{d}_{f}(H)$ is a closed subset of $H$ such that $\mathrm{d}_{f}(H) \subseteq$ $H^{1}(f, \varepsilon)$ for any $\varepsilon>0$.

Lemma 6.3. Suppose that $\alpha<\omega_{1}, \delta>0$ and $s>2$. If $x \in\left[K \backslash K^{1}(g, 1)\right]$ $\cap K^{\alpha}(f h, \delta)$, then

$$
x \in K^{\alpha}\left(f, \frac{\delta}{s(|h(x)|+1)} \wedge 1\right) .
$$

Proof. The proof is by induction on $\alpha$. For brevity, set

$$
K_{s, x}^{\alpha}=K^{\alpha}\left(f, \frac{\delta}{s(|h(x)|+1)} \wedge 1\right) .
$$

The result is clear if $\alpha=0$ or a limit ordinal. Assume that the lemma holds for some $\alpha<\omega_{1}$. Suppose $\delta>0$ and $s>2$ are given. Let $x \in$ $\left[K \backslash K^{1}(g, 1)\right] \cap K^{\alpha+1}(f h, \delta)$. If $x \in \mathrm{d}_{f}\left(K_{s, x}^{\alpha}\right)$, then $x \in K_{s, x}^{\alpha+1}$ and we are done. Otherwise, assume that $x \notin \mathrm{d}_{f}\left(K_{s, x}^{\alpha}\right)$. Then there exist a neighborhood $U_{1}$ of $x$ in $K$ and $M<\infty$ such that $|f(y)| \leq M$ for all $y \in U_{1} \cap K_{s, x}^{\alpha}$. Since $h=g_{0}$ on $K \backslash K^{1}(g, 1)$, and $g_{0}$ is continuous on $K \backslash K^{1}(g, 1)$, there exists a neighborhood $U_{2}$ of $x$ in $K$ such that $\left|h\left(x_{1}\right)-h\left(x_{2}\right)\right| \leq \delta /(2 M)$ and $2\left(\left|h\left(x_{1}\right)\right|+1\right)<s(|h(x)|+1)$ for all $x_{1}, x_{2} \in U_{2}$. Set $U=\left(U_{1} \cap U_{2}\right) \backslash K^{1}(g, 1)$. Then $U$ is a neighborhood of $x$.

Claim. $K^{\alpha}(f h, \delta) \cap U \subseteq K_{s, x}^{\alpha}$.

To see this, note that if $y \in U$, then $y \in U_{2}$. Hence there exists $t>2$ such that $t(|h(y)|+1) \leq s(|h(x)|+1)$. Also, $y \in K^{\alpha}(f h, \delta) \cap U$ implies that $y \in\left[K \backslash K^{1}(g, 1)\right] \cap K^{\alpha}(f h, \delta)$. Thus $y \in K_{t, y}^{\alpha}$ by the inductive hypothesis. Since

$$
\frac{\delta}{t(|h(y)|+1)} \wedge 1 \geq \frac{\delta}{s(|h(x)|+1)} \wedge 1
$$

we have $y \in K_{s, x}^{\alpha}$, as required.

Now if $V$ is a neighborhood of $x$ in $K$, then there exist $x_{1}, x_{2} \in U \cap V$ $\cap K^{\alpha}(f h, \delta)$ such that

$$
\begin{aligned}
\delta & \leq\left|f\left(x_{1}\right) h\left(x_{1}\right)-f\left(x_{2}\right) h\left(x_{2}\right)\right| \\
& \leq\left|f\left(x_{1}\right)-f\left(x_{2}\right)\right|\left|h\left(x_{1}\right)\right|+\left|h\left(x_{1}\right)-h\left(x_{2}\right)\right|\left|f\left(x_{2}\right)\right| \\
& \leq\left|f\left(x_{1}\right)-f\left(x_{2}\right)\right|\left|h\left(x_{1}\right)\right|+\frac{\delta}{2 M} \cdot M,
\end{aligned}
$$

where, in the last inequality, $\left|f\left(x_{2}\right)\right| \leq M$ since $x_{2} \in U \cap K_{s, x}^{\alpha}$ by the claim. Therefore, 


$$
\left|f\left(x_{1}\right)-f\left(x_{2}\right)\right| \geq \frac{\delta}{s(|h(x)|+1)} \wedge 1 .
$$

By the claim, $x_{1}, x_{2} \in V \cap K_{s, x}^{\alpha}$. Since $V$ is an arbitrary neighborhood of $x$, this shows that $x \in K_{s, x}^{\alpha+1}$ and completes the induction.

It follows from Lemma 6.3 that

$$
K^{\omega^{\xi_{1}}}(f h, \delta) \subseteq K^{1}(g, 1)
$$

Repeating the argument in Lemma 6.3 inductively yields

Lemma 6.4. $K^{\omega^{\xi_{1} \cdot \alpha}}(f h, \delta) \subseteq K^{\alpha}(g, 1)$ for all $\alpha<\omega_{1}$ and $\delta>0$.

In particular, $K^{\omega^{\xi_{1}} \cdot \omega^{\xi_{2}}}(f h, \delta)=\emptyset$ for all $\delta>0$, i.e., $f h \in \mathfrak{B}_{1}^{\xi_{1}+\xi_{2}}(K)$.

Theorem 6.5. If $f \in \mathfrak{B}_{1}^{\xi_{1}}(K)$ and $g \in \mathfrak{B}_{1}^{\xi_{2}}(K)$, then $f g \in \mathfrak{B}_{1}^{\xi}(K)$, where $\xi=\max \left\{\xi_{1}+\xi_{2}, \xi_{2}+\xi_{1}\right\}$.

Proof. From the above, we obtain a function $h$ on $K$ such that $\|g-h\|$ $\leq 1, \beta(h) \leq \omega^{\xi_{2}}$ and $f h \in \mathfrak{B}_{1}^{\xi_{1}+\xi_{2}}(K)$. Since $g, h \in \mathfrak{B}_{1}^{\xi_{2}}(K)$, it follows from Proposition 4.4 that $g-h \in \mathfrak{B}_{1}^{\xi_{2}}(K)$. As $g-h$ is bounded, we see that $g-h \in \mathcal{B}_{1}^{\xi_{2}}(K)$. By Lemma $6.2,(g-h) f \in \mathfrak{B}_{1}^{\xi_{2}+\xi_{1}}(K) \subseteq \mathfrak{B}_{1}^{\xi}(K)$. Also, $f h \in \mathfrak{B}_{1}^{\xi_{1}+\xi_{2}}(K) \subseteq \mathfrak{B}_{1}^{\xi}(K)$. Applying Proposition 4.4 again gives $f g=$ $f(g-h)+f h \in \mathfrak{B}_{1}^{\xi}(K)$.

Theorem 6.9 below shows that the result is sharp. First we show a strong result in this direction on spaces of ordinals. For $\alpha<\omega_{1}$, denote the ordinal interval $\left[0, \omega^{\alpha}\right]$ by $I_{\alpha}$.

Proposition 6.6. Let $0<\alpha, \xi_{1}<\omega_{1}$, and $h: I_{\omega^{\xi_{1} \cdot \alpha}} \rightarrow \mathbb{R}$ be a bounded function. Then there exist $f, g: I_{\omega} \xi_{1 \cdot \alpha} \rightarrow \mathbb{R}$ so that

(a) $\beta(f) \leq \omega^{\xi_{1}}$,

(b) $g$ takes values in $\mathbb{N}$,

(c) $g\left(\omega^{\omega^{\xi_{1}} \cdot \alpha}\right)=1$,

(d) $\left(I_{\omega \xi_{1 \cdot \alpha}}\right)^{\alpha}(g, \varepsilon) \subseteq\left\{\omega^{\omega^{\xi_{1} \cdot \alpha}}\right\}$ for all $\varepsilon>0$,

(e) $f g=h$.

The proof of Proposition 6.6 is postponed to the end of the section. The next two lemmas allow us to transplant the result onto general compact metric spaces.

Lemma 6.7. Let $K$ be a compact metric space. If $x_{0} \in K^{(\alpha)}$ for some $\alpha<\omega_{1}$, then there is a countable compact subspace $H \subseteq K$ such that $\left\{x_{0}\right\}=$ $H^{(\alpha)}$.

Proof. The proof is by induction on $\alpha$. The result is obvious if $\alpha=0$.

Suppose that the lemma is true for some $\alpha$. If $x_{0} \in K^{(\alpha+1)}$, then there exists a sequence $\left(x_{n}\right)$ in $K^{(\alpha)}, x_{n} \neq x_{0}$ for all $n$, that converges to $x_{0}$. We 
may also find a sequence $\left(U_{n}\right)$ of open sets with disjoint closures so that $x_{n} \in U_{n}$ for all $n \in \mathbb{N}$ and $\operatorname{diam}\left(U_{n}\right) \rightarrow 0$. Note that $x_{n} \in K^{(\alpha)} \cap U_{n} \subseteq$ $\left(\bar{U}_{n}\right)^{(\alpha)}$. Therefore by the inductive hypothesis, for all $n \in \mathbb{N}$, there is a countable compact set $H_{n} \subseteq \bar{U}_{n}$ such that $\left(H_{n}\right)^{(\alpha)}=\left\{x_{n}\right\}$. It is clear that $H=\left\{x_{0}\right\} \cup \bigcup_{n=1}^{\infty} H_{n}$ is a countable compact set such that $H^{(\alpha+1)}=\left\{x_{0}\right\}$.

Suppose the lemma is true for all $\alpha^{\prime}<\alpha$, where $\alpha<\omega_{1}$ is a limit ordinal. If $x_{0} \in K^{(\alpha)}$, then there exist a sequence $\left(\alpha_{n}\right)_{n=0}^{\infty}$ of ordinals that strictly increases to $\alpha$, a sequence $\left(x_{n}\right)$ converging to $x_{0}$ and a sequence $\left(U_{n}\right)$ of open sets with disjoint closures so that $x_{n} \in\left(\bar{U}_{n}\right)^{\left(\alpha_{n}\right)}$ for all $n \in \mathbb{N}$ and $\operatorname{diam}\left(U_{n}\right) \rightarrow 0$. By the inductive hypothesis, for all $n \in \mathbb{N}$, there is a countable compact set $H_{n} \subseteq \bar{U}_{n}$ such that $\left(H_{n}\right)^{\left(\alpha_{n}\right)}=\left\{x_{n}\right\}$. It is clear that $H=\left\{x_{0}\right\} \cup \bigcup_{n=1}^{\infty} H_{n}$ is a countable compact set such that $H^{(\alpha)}=\left\{x_{0}\right\}$.

Lemma 6.8. Let $K$ be a compact metric space. If $K^{(\alpha)} \neq \emptyset$ for some $0<\alpha<\omega_{1}$, then there is a subspace $L \subseteq K$ such that $L$ is homeomorphic to $I_{\alpha}$.

Proof. Suppose that $x_{0} \in K^{(\alpha)}$. By Lemma 6.7, there is a countable compact set $H$ such that $H^{(\alpha)}=\left\{x_{0}\right\}$. Since $H$ is countable and compact, by a theorem of Mazurkiewicz and Sierpiński (see, e.g., [5, Theorem 8.6.10]), $H$ is homeomorphic to an ordinal interval $[0, \beta]$. Since $H^{(\alpha)}=\left\{x_{0}\right\}$, it follows that $\omega^{\alpha} \leq \beta<\omega^{\alpha} \cdot 2$. Therefore $H$ is homeomorphic to $\left[0, \omega^{\alpha}\right]=I_{\alpha}$.

A consequence of Proposition 6.6 and Lemma 6.8 is the following.

Theorem 6.9. Suppose that $\xi_{1}, \xi_{2}$ are countable ordinals, and let

$$
\xi=\max \left\{\xi_{1}+\xi_{2}, \xi_{2}+\xi_{1}\right\} .
$$

If $K$ is a compact metric space such that $K^{(\xi)} \neq \emptyset$, then

$$
\sup \left\{\beta(f g): f \in \mathfrak{B}_{1}^{\xi_{1}}(K), g \in \mathfrak{B}_{1}^{\xi_{2}}(K)\right\}=\omega^{\xi} .
$$

Proof. We may of course assume that neither $\xi_{1}$ nor $\xi_{2}$ is 0 , and that $\xi=\xi_{1}+\xi_{2}$. Let $0<\alpha<\omega^{\xi_{2}}$. The assumption on $K$ yields a subspace $H \subseteq K$ that is homeomorphic to $I_{\omega \xi_{1} \cdot \alpha}$ (Lemma 6.8) and a $\{0,1\}$-valued function $h$ in $\mathfrak{B}_{1}(H)$ such that $H^{\omega^{\xi_{1} \cdot \alpha}}(h, 1) \neq \emptyset$. Applying Proposition 6.6 to $h: H \rightarrow \mathbb{R}$, we obtain $f, g: H \rightarrow \mathbb{R}$ with properties as given in the proposition. Extend $f, g$, and $h$ to $K$ by defining them to be 0 on $K \backslash H$. Then $\beta_{K}(h) \geq \beta_{H}(h) \geq \omega^{\omega^{\xi_{1}} \cdot \alpha}$. Also, $K^{1}(f, \varepsilon) \subseteq H$ for all $\varepsilon>0$. Hence $K^{1+\omega^{\xi_{1}}}(f, \varepsilon) \subseteq H^{\omega^{\xi_{1}}}(f, \varepsilon)=\emptyset$. Therefore $\beta(f) \leq \omega^{\xi_{1}}$. Likewise, $\beta(g) \leq 1+$ $\alpha+1 \leq \omega^{\xi_{2}}$. Summarizing, we have functions $f$ and $g$ such that $f \in \mathfrak{B}_{1}^{\xi_{1}}(K)$, $g \in \mathfrak{B}_{1}^{\xi_{2}}(K)$ and $\beta(f g) \geq \omega^{\xi_{1}} \cdot \alpha$. Since $\alpha<\omega^{\xi_{2}}$ is arbitrary, the theorem is proved.

Proof of Proposition 6.6. The proof is by induction on $\alpha$. Let $h: I_{\omega \xi_{1 \cdot \alpha}}$ $\rightarrow \mathbb{R}$ be a function so that $|h|<M$ for some $M>0$. Choose a sequence 
$\left(\tau_{k}\right)_{k=0}^{\infty}$ of ordinals with $\tau_{0}=0$ that strictly increases to $\omega^{\xi_{1}}$. Define a function $G: I_{\omega \xi_{1}} \rightarrow \mathbb{R}$ by

$$
G(t)= \begin{cases}k & \text { if } \omega^{\tau_{k-1}}<t \leq \omega^{\tau_{k}}, k \in \mathbb{N}, \\ 1 & \text { if } t=0,1, \text { or } \omega^{\omega^{\xi_{1}}} .\end{cases}
$$

If $\alpha=1$, let $g=G$ and $f=h / g$. Note that $g$ is constant on each of the open sets $\left(\omega^{\tau_{k-1}}, \omega^{\tau_{k}}\right]$. Therefore, for all $\varepsilon>0,\left(I_{\omega \xi_{1}}\right)^{1}(g, \varepsilon) \subseteq\left\{\omega^{\omega^{\xi_{1}}}\right\}$. If $t_{1}, t_{2}>$ $\omega^{\tau_{k-1}}$, then $\left|f\left(t_{1}\right)-f\left(t_{2}\right)\right|<2 M / k$. This implies that $\left(I_{\omega} \xi_{1}\right)^{1}(f, 2 M / k) \subseteq$ $\left[0, \omega^{\tau_{k-1}}\right] \cup\left\{\omega^{\omega^{\xi_{1}}}\right\}$. Hence $\left(I_{\omega_{\xi_{1}}}\right)^{1+\tau_{k-1}+1}(f, 2 M / k) \subseteq\left[0, \omega^{\tau_{k-1}}\right]^{\left(\tau_{k-1}+1\right)}=\emptyset$. Thus $\left(I_{\omega \xi_{1}}\right)^{\omega^{\xi_{1}}}(f, 2 M / k)=\emptyset$ for all $k$, which means that $\beta(f) \leq \omega^{\xi_{1}}$.

Suppose that the proposition is true for some $\alpha$. For each $\lambda<\omega^{\omega^{\xi_{1}}}$, let $J_{\lambda}$ denote the clopen ordinal interval $\left(\omega^{\omega^{\xi_{1}} \cdot \alpha} \cdot \lambda, \omega^{\omega^{\xi_{1}} \cdot \alpha} \cdot(\lambda+1)\right]$. Since $J_{\lambda}$ is homeomorphic to $I_{\omega \xi_{1 \cdot \alpha}}$ for all $\lambda<\omega^{\omega^{\xi_{1}}}$, by the inductive hypothesis, there are functions $f_{\lambda}, g_{\lambda}: J_{\lambda} \rightarrow \mathbb{R}$ such that

(a) $\beta\left(f_{\lambda}\right) \leq \omega^{\xi_{1}}$,

(b) $g_{\lambda}$ takes values in $\mathbb{N}$,

(c) $g_{\lambda}\left(\omega^{\omega^{\xi_{1}} \cdot \alpha} \cdot(\lambda+1)\right)=1$,

(d) $\left(J_{\lambda}\right)^{\alpha}\left(g_{\lambda}, \varepsilon\right) \subseteq\left\{\omega^{\omega^{\xi_{1} \cdot \alpha}} \cdot(\lambda+1)\right\}$ for all $\varepsilon>0$,

(e) $f_{\lambda} g_{\lambda}=h_{\mid J_{\lambda}}$.

Define $g: I_{\omega^{\xi_{1} \cdot(\alpha+1)}} \rightarrow \mathbb{R}$ by

$$
g(t)= \begin{cases}G(\lambda) g_{\lambda}(t) & \text { if } t \in J_{\lambda}, 0 \leq \lambda<\omega^{\omega^{\xi_{1}}}, \\ 1 & \text { if } t=0 \text { or } \omega^{\omega^{\xi_{1} \cdot(\alpha+1)}}\end{cases}
$$

and let $f=h / g$. It suffices to verify properties (a) and (d). For each $\lambda<\omega^{\omega^{\xi_{1}}}$,

$$
\begin{aligned}
& \left(I_{\omega}^{\xi_{1} \cdot(\alpha+1)}\right)^{\alpha}(g, \varepsilon) \cap J_{\lambda} \subseteq\left(J_{\lambda}\right)^{\alpha}\left(G(\lambda) g_{\lambda}, \varepsilon\right) \quad \text { by Lemma 2.1(c) } \\
& =\left(J_{\lambda}\right)^{\alpha}\left(g_{\lambda}, \varepsilon / G(\lambda)\right) \subseteq\left\{\omega^{\omega^{\xi_{1} \cdot \alpha}} \cdot(\lambda+1)\right\} .
\end{aligned}
$$

Therefore

$$
\left(I_{\omega \xi_{1} \cdot(\alpha+1)}\right)^{\alpha}(g, \varepsilon) \subseteq\left\{\omega^{\omega^{\xi_{1}} \cdot \alpha} \cdot \lambda: \lambda \leq \omega^{\omega^{\xi_{1}}}\right\} .
$$

Using the fact that $g\left(\omega^{\omega^{\xi_{1}} \cdot \alpha} \cdot(\lambda+1)\right)=G(\lambda)$ for all $\lambda<\omega^{\omega^{\xi_{1}}}$ and the fact, proved above, that $\left(I_{\omega \xi_{1}}\right)^{1}(G, \varepsilon) \subseteq\left\{\omega^{\omega^{\xi_{1}}}\right\}$, we see that $\left(I_{\omega^{\xi_{1}} \cdot(\alpha+1)}\right)^{\alpha+1}(g, \varepsilon)$ $\subseteq\left\{\omega^{\omega^{\xi_{1}} \cdot(\alpha+1)}\right\}$.

Now consider $f$. Note that on $J_{\lambda}, f=h / g=h /\left(G(\lambda) g_{\lambda}\right)=f_{\lambda} / G(\lambda)$ and that $G(\lambda) \geq 1$. As a result,

$$
\left(I_{\omega \xi_{1} \cdot(\alpha+1)}\right)^{\omega^{\xi_{1}}}(f, \varepsilon) \cap J_{\lambda} \subseteq\left(J_{\lambda}\right)^{\omega^{\xi_{1}}}\left(f_{\lambda}, \varepsilon\right)=\emptyset .
$$

Since $|f|<M / G(\lambda)$ on $J_{\lambda}$, we have $|f|<M / k$ on $\bigcup\left\{J_{\lambda}: \omega^{\tau_{k-1}}<\lambda<\omega^{\omega^{\xi_{1}}}\right\}$. Hence 


$$
\left(I_{\omega^{\xi_{1} \cdot(\alpha+1)}}\right)^{1}(f, 2 M / k) \subseteq \bigcup\left\{J_{\lambda}: \lambda \leq \omega^{\tau_{k-1}}\right\} \cup\left\{\omega^{\omega^{\xi_{1} \cdot(\alpha+1)}}\right\} .
$$

In particular, $\omega^{\omega^{\xi_{1}} \cdot(\alpha+1)} \notin\left(I_{\omega \xi_{1} \cdot(\alpha+1)}\right)^{2}(f, 2 M / k)$ for all $k \in \mathbb{N}$. This fact together with (6.1) implies that $\left(I_{\omega \xi_{1} \cdot(\alpha+1)}\right)^{\omega^{\xi_{1}}}(f, \varepsilon)=\emptyset$ for all $\varepsilon>0$. This proves the proposition for $\alpha+1$.

Suppose $\alpha<\omega_{1}$ is a limit ordinal and the statement holds for all ordinals $\alpha^{\prime}<\alpha$. Choose a sequence of ordinals $\left(\eta_{k}\right)$ with $\eta_{0}=0$ that strictly increases to $\alpha$. For each $k \in \mathbb{N}$, let $L_{k}$ be the ordinal interval $\left(\omega^{\omega^{\xi_{1}} \cdot \eta_{k-1}}, \omega^{\omega^{\xi_{1}} \cdot \eta_{k}}\right]$, which is homeomorphic to $I_{\omega} \xi_{1} \cdot \eta_{k}$. By the inductive hypothesis, for each $k \in \mathbb{N}$, there are $f_{k}, g_{k}: L_{k} \rightarrow \mathbb{R}$ such that

(a) $\beta\left(f_{k}\right) \leq \omega^{\xi_{1}}$,

(b) $g_{k}$ takes values in $\mathbb{N}$,

(c) $g_{k}\left(\omega^{\omega^{\xi_{1}} \cdot \eta_{k}}\right)=1$,

(d) $\left(L_{k}\right)^{\eta_{k}}\left(g_{k}, \varepsilon\right) \subseteq\left\{\omega^{\omega^{\xi_{1}} \cdot \eta_{k}}\right\}$,

(e) $f_{k} g_{k}=h_{\mid L_{k}}$.

Define $g: I_{\omega^{\xi_{1} \cdot \alpha}} \rightarrow \mathbb{N}$ by

and set $f=h / g$. Clearly,

$$
g(t)= \begin{cases}k g_{k}(t), & t \in L_{k}, \\ 1, & t=0,1, \text { or } \omega^{\omega^{\xi_{1}} \cdot \alpha},\end{cases}
$$

$$
\begin{aligned}
\left(I_{\omega \xi_{1} \cdot \alpha}\right)^{\eta_{k}}(g, \varepsilon) \cap L_{k} & \subseteq\left(L_{k}\right)^{\eta_{k}}\left(g_{k}, \varepsilon\right) \quad \text { by Lemma 2.1(c) } \\
& \subseteq\left\{\omega^{\omega^{\xi_{1} \cdot \eta_{k}}}\right\} .
\end{aligned}
$$

Hence

$$
\left(I_{\omega \xi_{1} \cdot \alpha}\right)^{\alpha}(g, \varepsilon) \subseteq\left\{\omega^{\omega^{\xi_{1} \cdot \alpha}}\right\}
$$

Since $|h|<M$, we have $|f(t)|<M / k$ whenever $t \in \bigcup_{j=k}^{\infty} L_{j}$. Therefore

$$
\left(I_{\omega \xi_{1} \cdot \alpha}\right)^{1}(f, 2 M / k) \subseteq \bigcup_{j=1}^{k-1} L_{j} \cup\left\{\omega^{\omega^{\xi_{1} \cdot \alpha}}\right\} .
$$

Since $\omega^{\omega^{\xi_{1}} \cdot \alpha}$ is an isolated point in $\bigcup_{j=1}^{k-1} L_{j} \cup\left\{\omega^{\omega^{\xi_{1}} \cdot \alpha}\right\}$, it follows that $\omega^{\omega^{\xi_{1}} \cdot \alpha} \notin\left(I_{\omega \xi_{1} \cdot \alpha}\right)^{2}(f, 2 M / k)$ for all $k \in \mathbb{N}$. Finally, $\left(I_{\omega \xi_{1} \cdot \alpha}\right)^{\omega^{\xi_{1}}}(f, 2 M / k) \cap$ $L_{k} \subseteq\left(L_{k}\right)^{\omega^{\xi_{1}}}(f, 2 M / k)=\emptyset$. Hence $\left(I_{\omega \xi_{1} \cdot \alpha}\right)^{\omega^{\xi_{1}}}(f, \varepsilon)=\emptyset$ for all $\varepsilon>0$. This completes the induction.

\section{References}

[1] J. Dugundji, Topology, Allyn and Bacon, Boston, 1966.

[2] R. Haydon, E. Odell and H. P. Rosenthal, On certain classes of Baire-1 functions with applications to Banach space theory, in: Functional Analysis (Austin, TX, 1987-89), Lecture Notes in Math. 1470, Springer, New York, 1991, 1-35. 
[3] A. S. Kechris and A. Louveau, A classification of Baire class 1 functions, Trans. Amer. Math. Soc. 318 (1990), 209-236.

[4] P. Kiriakouli, A classification of Baire-1 functions, ibid. 351 (1999), 4599-4609.

[5] Z. Semadeni, Banach Spaces of Continuous Functions, Vol. 1, Polish Sci. Publ., Warszawa, 1971.

Denny H. Leung

Department of Mathematics

National University of Singapore

2 Science Drive 2, Singapore 117543

E-mail: matlhh@nus.edu.sg
Wee-Kee Tang Mathematics and Mathematics Education National Institute of Education Nanyang Technological University 1 Nanyang Walk, Singapore 637616 E-mail: wktang@nie.edu.sg

Received 30 January 2003;

in revised form 6 November 2003 